\title{
Reactive Synaptogenesis and Neuron Densities for Neuropeptide Y, Somatostatin, and Glutamate Decarboxylase Immunoreactivity in the Epileptogenic Human Fascia Dentata
}

\author{
Gary W. Mathern, ${ }^{1}$ Thomas L. Babb, ${ }^{2,3}$ James K. Pretorius, ${ }^{3}$ and Joao P. Leite ${ }^{3}$ \\ ${ }^{1}$ Divisions of Neurosurgery and Clinical Neurophysiology, ${ }^{2}$ Department of Neurology, and ${ }^{3}$ Brain Research Institute, \\ UCLA School of Medicine, Los Angeles, California 90024-8461
}

This study determined differences of fascia dentata (FD) peptide and inhibitory neuroanatomy between patients with epileptogenic hippocampal sclerosis (HS), those with extrahippocampal seizure pathologies, and autopsy comparisons. Surgically treated temporal lobe epilepsy patients were clinically classified into two pathogenic categories: (1) HS with focal mesial temporal neuroimaging and histories of initial precipitating injuries to the brain $(n=18)$ and (2) non-HS patients with extrahippocampal mass lesions or idiopathic seizures (i.e., without lesions or HS; mass lesion/ldiopathic; $\boldsymbol{n}=9$ ). The hippocampal sections were studied for (1) granule cell, hilar, CA4, and CA3 neuron densities; (2) hilar densities and the percentage of neurons immunoreactive (IR) for neuropeptide Y (NPY), somatostatin (SS), and glutamate decarboxylase (GAD); (3) densities of GAD neurons in the lower granule cell and infragranular zone (basket-like cells); (4) the semiquantitative pattern of IR peptides/GAD FD molecular layer axon sprouting; (5) IR gray values (GV) of the FD molecular layers; and (6) the thickness of the supragranular molecular layer.

Results showed the following. (1) Compared to autopsies, both HS and mass lesion/idiopathic patients showed less granule cell and CA3 neuron densities, but there were no statistical differences between the latter two pathogenic categories. (2) By contrast, compared to autopsies and mass lesion/idiopathic cases, HS patients showed less hilar and CA4 neuron densities, and there were no differences between autopsies and mass lesion/idiopathic. (3) Compared to autopsies, the NPY and SS hilar neuron densities in HS patients, but not mass lesion/idiopathic cases, were less. (4) Compared to autopsies, the hilar GAD neuron densities for HS and mass lesion/idiopathic patients were not less. (5) In HS patients the averaged percentages of hilar SS neurons were less than autopsies, and no other differences of IR hilar percentages were found. (6) The densities of GAD basket-like neurons and the thickness of the supragranular molecular layer were not different between

\footnotetext{
Received Aug. 16, 1994; revised Jan. 4, 1994; accepted Jan. 12, 1994.

This work was supported by NIH Grant NS 02808. a Clinical Investigator Development Award to G.W.M. (K08 NS 1603), and a Fogarty Fellowship to J.P.L. (F05 TW04959). Barbara G. Vickrey, M.D., generously provided followup data on seizure outcomes on some of the patients and was supported by AHCPR Grant RO) HSO6856.

Correspondence should be addressed to Gary W. Mathern, M.D., Division of Neurosurgery, Reed Neurological Research Center, UCLA Medical Center, Los Angeles, CA 90024-1769.

Copyright 1995 Society for Neuroscience $0270-6474 / 95 / 153990-15 \$ 05.00 / 0$
}

any combination of pathogenic categories and autopsies. (7) By semiquantitative visual assessments, peptides/GAD axon sprouting into the FD was greater in HS compared to mass lesion/idiopathic or autopsies. (8) Compared to mass lesion/idiopathic cases, in HS NPY outer molecular layer GVs were lower, SS GVs were not different, and GAD inner molecular layer GVs were higher. (9) Analyses comparing the two pathogenic categories and neuron densities with peptides/GAD axon sprouting found six comparisons that correlated sprouting with hilar and CA4 neuron losses, and four comparisons showing greater sprouting in HS compared to mass lesion/idiopathic.

These data indicate that there were greater hilar and CA4 neuron losses and IR axon sprouting for NPY, SS, and GAD in the epileptogenic human fascia dentata compared to mass lesion/idiopathic patients with extrahippocampal seizures, and autopsies. Further, measures of IR sprouting correlated with hilar neuron losses, and hilar and CA4 neuron losses along with peptides/GAD axon sprouting were associated with clinical histories of initial precipitating injuries to the brain. This supports the notion that in HS reorganized NPY, SS, and GAD axon circuits, which are possibly inhibitory, may reinnervate the dendrites of granule cells following hippocampal injury and hyperinnervate the proximal dendritic zone, contributing to the pathophysiology of human hippocampal seizures.

[Key words: hippocampal sclerosis, tumors, complex partial seizures, peptides, synaptic reorganization, inhibition]

Studies of human temporal lobe epilepsy (TLE) over the past several decades have found several important pathophysiologic associations. For example, in surgically treated patients the most frequent pathologic findings are areas of focal pathology. The most common are (1) severe hippocampal neuron losses and gliosis, termed hippocampal sclerosis (HS), and (2) mass lesions (Babb et al., 1984b; Babb and Brown, 1987; Babb, 1991; Mathcrn ct al., 1995a). By contrast, autopsies of patients with various antemortem seizure types show less and more variable amounts of hippocampal neuron damage and this most likely represents extrahippocampal seizure associated damage rather than neuron losses associated with epileptogenesis (Margerison and Corsellis, 1966; Babb, 1991; Mathern et al., 1995c,d). In surgically treated cases, averaged neuron densities are lower in HS compared to mass lesions (Babb and Brown, 1987; Mathern et al., 1995a), and intracerebral electrodes recordings indicate that HS 
is strongly associated with the chronic epileptogenic region while, by contrast, in mass lesions seizures probably propagate into the hippocampus (Babb et al., 1984a, 1987; Spencer et al., 1993). Further, post-resection seizure control is more frequent in patients with focal pathologies compared to idiopathic TLE patients (i.e., without hippocampal or temporal pathology), supporting the notion that the epileptic focus in the former patients was removed (Mathern et al., 1995b,d). Conceptually, it is not difficult to accept the notion that temporal mass lesions interacting with surrounding brain including the hippocampus participates in the pathogenesis of seizures. However, it is a paradox that an area of severe neuron losses (HS) can be epileptogenic. Over the last decade several hypotheses have been suggested, many directed at the fascia dentata for its role as the physiologic "gatekeeper" into the hippocampus, and the proposed pathophysiologic mechanisms have primarily concentrated on alterations in excitation and/or inhibition (Anderson et al., 1966; Sloviter, 1989; Babb et al., 1991; Mathern et al., 1993).

Currently, there are two principle hypotheses debated in the literature concerning fascia dentata epileptogenesis. The first has been based on rat perforant path stimulation experiments (Sloviter and Damiano, 1981; McNamara, 1994; Sloviter, 1987, 1989, 1994). This model showed that with short periods of perforant path stimulation there were no qualitative signs of hippocampal neuron injury and even with longer periods of stimulation (over 8-12 hr) the first neurons excited, the granule cells (GC), were not damaged. However, with periods of stimulation that approached $24 \mathrm{hr}$ there was neuron damage first to hilar neurons followed by CA3 pyramids. The damage after $24 \mathrm{hr}$ of stimulation has been equated to human "end folium sclerosis" (Sloviter, 1987, 1989) which as a pathologic diagnosis is uncommon in surgically treated patients (Bruton, 1988; Babb, 1991). Extracellular GC recordings from those rats with perforant path induced damage showed short and long term loss of paired pulse inhibition and multiple GC population spikes (Sloviter, 1987). It was initially presumed that the electrophysiologic data represented a decrease in inhibition rather than an increase in excitation, and was from excitotoxic loss of fascia dentata GABAergic neurons (Ribak et al., 1979, 1989; Sloviter, 1983). However, subsequent qualitative anatomic studies of the model showed preservation of GABA basket cells in the infragranular zone and preserved GABAergic axons around GCs, similar to the findings in the rat kainate model and human hippocampal sclerosis (Babb et al., 1989; Davenport et al., 1990). Instead, there was loss of hilar somatostatin (SS) and neuropeptide Y (NPY) neurons as well as additional hilar damage consistent with mossy cell losses (Sloviter, 1987, 1989).

From these rat studies the dormant basket cell hypothesis was proposed to explain the neuropathology, pathogenesis, and pathophysiology of the rat model and human hippocampal seizures (McNamara, 1994; Sloviter, 1994). It states that following hippocampal seizures there is loss of "inhibitory" hilar SS and NPY neurons. To further explain the loss of physiologic inhibition, the hypothesis proposed that surviving GABA basket cells are physiologically "dormant" due to loss of afferent synaptic connections from destroyed hilar mossy cells (Sloviter, 1987, 1989, 1991, 1994). Moreover, loss of physiologic inhibition is an immediate and permanent feature following hippocampal seizure associated hilar neuron losses and precedes axon reorganization and sprouting (Sloviter, 1992, 1994). Hence, the proposed pathogenesis of HS consists of a cycle whereby any first seizure damages hilar neurons, lowering inhibition and gen- erating spontaneous subclinical seizures because of the decreased thresholds. Each additional subclinical seizure further damages the hippocampus, repeating the cycle until the patient presents with the clinical TLE seizure syndrome. While this hypothetical scheme ties together the physiologic and anatomic data from the perforant path rat model, it is unclear if human epileptogenic hippocampi demonstrate the neuroanatomic changes predicted by the rat model and the proposed pathogenic hypothesis. Further, it is not known if the expected anatomic changes are also found in human hippocampi that are not epileptogenic but are involved in extrahippocampal temporal seizure activity (i.e., tumors) which may be similar to the excitotoxic perforant path stimulation model.

The second current pathophysiologic hypothesis is one of synaptic reorganization. It states that after an initial precipitating injury (IPI) that preferentially damages hilar along with other principal hippocampal cells, surviving fascia dentata neurons respond by sprouting axon collaterals to reinnervate deafferented synapses, and the new synaptically reorganized axon synapses form aberrant circuits and connections that contribute to epileptogenesis (Babb et al., 1991, 1992; de Lanerolle et al., 1992; Mathern et al., 1993, 1995a). Animal studies have shown that hippocampal axons including mossy fibers, and GABAergic fibers sprout and reinnervate deafferented synapses following neuron injury (Laurberg and Zimmer, 1981; Smialowski and Smialowski, 1981; Tauck and Nadler, 1985; Davenport et al., 1990; Mathern et al., 1992). Reorganized axons, especially mossy fibers, have been associated with spontaneous chronic hippocampal seizures (Cavalheiro et al., 1982; Mathern et al., 1993), and mossy fiber sprouting into the fascia dentata inner molecular layer has been demonstrated in humans (Babb et al., 1988b, 1991; de Lanerolle et al., 1989; Houser et al., 1990). There is greater fascia dentata mossy fiber sprouting in HS compared to mass lesion patients, and HS patients nearly always have clinical IPI histories (Mathern et al., 1994b, 1995a,b). Likewise, qualitative human studies indicate that in HS NPY and SS fibers seem to sprout in the fascia dentata (de Lanerolle et al., 1989, 1992; de Lanerolle and Spencer, 1991), but it is unclear if these labeled peptides show quantitative differences between epileptogenic hippocampi compared to patients with extrahippocampal seizures. Further, inhibitory glutamate decarboxylase (GAD) containing neurons around principal neurons and GAD puncta densities are spared in HS (Babb et al., 1989; Babb, 1992). However, it is unclear if there are increases in GAD fascia dentata sprouting that are specific pathologic markers associated with HS, and epileptogenesis similar to animal experiments (Davenport et al., 1990).

This study determined, in human surgically treated TLE patients, if there were quantitative differences between HS compared to non-HS patients for (1) fascia dentata and regio inferior neuron losses, (2) molecular layer axon sprouting for NPY and SS peptides, and (3) molecular layer inhibitory GAD fiber sprouting. The purpose and design was to discern if there was anatomic evidence of peptide and inhibitory axon re- or hyperinnervation associated with hilar neuron losses that were specific pathologic markers in the epileptogenic hippocampus. We tested the hypothesis that patients with epileptogenic hippocampi associated with IPI histories and sclerosis would show greater hilar neuron losses and synaptic reorganization for NPY, SS, and GAD fibers compared to mass lesions and idiopathic TLE patients without IPI histories whose seizures probably do not originate in the hippocampus. Further, we tested the hypothesis that 
molecular layer fascia dentata sprouting would correlate with hilar neuron losses. These hypotheses were tested on patients ( $n$ $=27$ ) from a single epilepsy center evaluated and treated using standardized clinical protocols and surgical procedures.

This laboratory has studied and tested similar hypotheses for human fascia dentata mossy fiber sprouting published in recent reports (Mathern et al., 1995a-c).

\section{Materials and Methods}

Clinical material. Patients with intractable complex partial scizures of probable temporal lobe origin were evaluated at UCLA's Clinical Neurophysiology Program (CNP) using standardized protocols previously published, the protocols were approved by the institution's Human Subject Protection Committee, and informed consent was obtained for use of any data for research studies (Engel et al., 1991; Engel, 1993). The diagnostic approach presumed that temporal lobe seizures began in or adjacent to regions of cerebral damage, and those regions could be localized based on the anatomic area of epileptic excitability and functional deficits. The decision for surgical therapy was based on localizing a single area for resection. Evaluation included detailed history and neurological examinations, interictal and ictal scalp/sphenoidal EEG, an extensive neuropsychological test battery, and intracarotid amobarbital injections (Wada test) for memory and speech representation. Neuroimaging studies included high-resolution MRI with special protocols to visualize the hippocampal formation and 18-fluoro-2-deoxyglucose (FDG) PET. If the initial (phase I) noninvasive data localized the focus to the anterior temporal region, patients were referred for a standardized en bloc resection including 3-4 cm of the hippocampus (Crandall, 1987; Skip patients; phase III). Alternately, if the initial data strongly suggested temporal lobe seizures but were insufficient to lateralize the lobe, then bilateral orthogonal temporal intracerebral electrodes were used to determine the epileptic lobe and area of temporal seizure onsets (Crandall et al., 1963; phase II; CNP patients).

There were a total of 51 temporal resections between December, 1988 to June, 1992 (32 Skip, 19 CNP) in which the pathologic specimens were of sufficient quality to study both the temporal neocortex and hippocampus. Of these cases, $27(53 \%)$ were used in this study. The lateral neocortcx was scrially sectioncd, microscopically cxamincd by the neuropathologist, all macroscopic mass lesions were identified, and pathologically classified.

Clinical data collection. For the purposes of this study we documented if patients had any medical events prior to their onset of temporal lobe epilepsy and neuroimaging studies that indicated probable hippocampal sclerosis before temporal resection. Such information was abstracted from the medical record, and we have previously defined these medical events as initial precipitating injuries (IPI; Mathern et al., $1994 \mathrm{~b}, 1995 \mathrm{~b}, \mathrm{~d})$. These data were collected in a uniform manner by one author (GWM) who used a standardized format for data collection. Each medical record was carefully reviewed with special attention to the multiple interviews each patient had with medical personnel as part of the CNP evaluations. Any medical event or cerebral injury was noted and designated as significant if it was associated with unconsciousness for more than $30 \mathrm{~min}$ or alteration in cognition for more than $4 \mathrm{hr}$. For example, a note in the medical record of a prior "febrile seizure" was insufficient. The interviewers' notes had to contain details of the injury (i.e., status epilepticus) and information on the patients condition after the injury (i.e., unconscious and hospitalized). Information was deemed reliable if similar data were obtained from separate notes by different interviewers. If details were lacking, attempts were made to contact the patient or a family member. These data along with neuroimaging data were collected without knowledge of the final hippocampal pathology and classified into the following categories. Hippocampal sclerosis (HS) were patients with an IPI history prior to the onset of their TLE and with evidence of focal mesial temporal anatomic and/or functional pathology on MRI or PET (i.e., hippocampal atrophy and/or PET hypometabolism). In this group the chronic temporal lobe seizures were presumed to arise from the mesial temporal structures based on the depth electrode investigations, ictal scalp EEG, and prior published pathophysiologic studies of similar patients (Babb et al., 1984a,b, 1987). Further, significant postresection seizure reduction or relief implied that the epileptogenic focus had been removed (Mathern et al., 1995d). Mass lesion patients usually do not have IPI histories, had verified macroscopic neuropathology, and presumably seizures originated primarily from the extrahippocampal lesion based on prior published studies (Goldring and Gregorie, 1986; Awad et al., 1991; Berger et al., 1993; Cascino et al., 1993). Idiopathic TLE patients were without IPI histories or mass lesions, there was very little post-resection seizure reduction, and hence there was not a pathophysiologic seizure focus in the resected anterior temporal lobe similar to previous reports (Mathern et al., $1995 \mathrm{~b}, \mathrm{~d})$. The patients with mass lesions and idiopathic TLE were cataloged into a non-HS group (mass lesion/idiopathic) based on the presumption that a component of their seizures began outside the hippocampus (Mathern et al., 1994a,b, 1995a,d).

Other clinical variables abstracted from the medical record were (1) age at the time of the IPI; (2) onset of spontaneous habitual TLE defined as that age when the patient's typical habitual seizures, for which they were referred for surgical consideration, were first recognized by physicians and/or family as distinct events; (3) age at surgery; and (4) side resected. For each HS patient, a latent period was determined defined as the interval in years from the IPI until the onset of their habitual TI.F. For all patients, the duration of habitual TLE was the interval between the onset of TLE until surgery (Mathern et al., 1994b, 1995d).

Patient follow-up was obtained using a standardized format abstractcd from the medical record and research files. This information was collected independently of the above clinical and pathologic information. Outcome was classified using a modification of the scale proposed by Engel (1987) and was based on the incidence of seizures for the most recent 12 month period. The number of years since surgery was also recorded. Groups were cataloged as completely seizure free with or without simple sensory seizures (auras; class 1), fewer than six seizures per year (class 2), between 0.5 to 2 seizures per month (class 3 ), and more than 2 seizures per month (class 4 ). The data was checked for accuracy for a subset of patients by comparison to a 1990 mailed survey that was collected and maintained separately from this study (Vickrey et al., 1992).

Tissue collection and immunocytochemical processing. Hippocampal specimens from HS and mass lesion/idiopathic patients were freshly collected in the operating room and a $1 \mathrm{~cm}$ block was fixed in phosphate buffered $4 \%$ paraformaldehyde. The ICC protocols were similar to previous publications (Babb et al., 1989; Mathern et al., 1994a). Antisera identified immunoreactivity (IR) for neuropeptide Y (NPY; 1:4000 dilution, from rabbit; Peninsula Laboratories, Inc., Belmont, CA), somatostatin 28 ( $\mathrm{SS}$; the 1-14 subunit; 1:2000 dilution, from rabbit; Incstar, Stillwater, Minnesota), and glutamate decarboxylase (GAD; 1:2000 dilution, from sheep; Oertel et al., 1981). Briefly, $30 \mu \mathrm{m}$ adjacent sections cut transverse to the axis of the hippocampus were collected from the cryostat and placed in individual $3 \mathrm{ml}$ wells containing $0.05 \mathrm{M}$ Tris$\mathrm{HCl}$ buffered saline (TBS, $\mathrm{pH} 7.6$ ), and free floating sections were processed the same day as follows, with 10 min TBS rimses (three changes) between each step: $5 \mathrm{~min}$ in 3\% hydrogen peroxide, $10 \%$ methanol in TBS; 60 min in a blocking solution of $1.5 \%$ normal serum in TBS; overnight in primary antisera diluted in $1 \%$ normal blocking serum; 35 min in diluted biotinylated species specific anti-Ig sera (goat anti-rabbit; rabbit anti-sheep; ABC kit, Vector Labs); and $60 \mathrm{~min}$ in a solution of excess avidin and biotinylated HRP (Vector Labs). The sections were developed simultaneously for 7-10 $\mathrm{min}$ in $0.05 \% 3,3^{\prime}$-diaminobenzidine tetrahydrochloride and $0.01 \%$ hydrogen peroxide. After sufficient colorization the reaction was halted by washing in several rinses of cold TBS, mounted on subbed slides, air dried, treated for $35 \mathrm{sec}$ in $0.1 \%$ osmium tetroxide in $0.1 \mathrm{M}$ phosphate buffer $(\mathrm{pH} \mathrm{7.2)}$, dehydrated through graded alcohols to xylene, and coverslipped. The tissue was processed within 3-4 d of surgery, and NPY, SS, and GAD were coprocessed to ensure uniform IR staining.

For comparison purposes, three human autopsies were processed and analyzed for IR to the two peptides and GAD. One autopsy patient was a 49 year old who died from idiopathic cardiomyopathy. The second was a 56 year old with metastatic adenocarcinoma, and the third was a 59 year old who died from an acute myocardial infarction. None of the patients had antemortem clinical seizures or evidence of brain pathology on postmortem examination. The specimens were collected within a few hours after death prior to autolysis, and processed in the same manner as the surgical cases.

Tissue analysis. NPY, SS, and GAD hippocampal sections were analyzed for the following. (1) The estimated density and percentage of all three IR neurons in the fascia dentata hilus. (2) The estimated density of GAD neurons in the lower stratum granulosum and infragranular zone consistent with inhibitory basket-like cells. (3) The average thickness (i.e., height) of the supragranular molecular layer. (4) A semiquan- 
titative visual assessment of the pattern of IR puncta and axon fiber sprouting in the fascia dentata molecular layer. (5) The IR gray values $(\mathrm{GV})$ of the density in the inner molecular layer (IML) compared to the middle and outer molecular layer. The middle and outer molecular layer in the remainder of the text will be collectively referred to as the "OML." All data were collected independently and blindly to the clinical and other pathologic data.

Density and percentage of hilar IR neurons. This method was similar to our prior published technique used to determine the estimated density of other fascia dentata neurons in the adjacent Nissl sections (see below). At $400 \times$ an ocular grid was positioned over the hilus. The pyramids of CA4 and CA3c located between the blades of the stratum granulosum and the polymorphic cells in the subgranular zone (a 100 $\mu \mathrm{m}$ thick area) were avoided by positioning the grid in areas that clearly contained only hilar neurons. Neurons with distinctly IR soma within the grid were counted, the grid moved to another hilar location and the procedure repeated until the entire hilar region was covered. Using Abercrombie's (1946) correction for nuclear diameter $(14 \mu \mathrm{m})$ and section thickness $(30 \mu \mathrm{m})$, the estimated density of IR neurons per cubic millimeter was determined. Finally, the relative percentage of hilar IR ncurons was calculated as the density of IR neurons divided by the density of total hilar neurons as measured from the adjacent Nissl section from that hippocampal specimen.

Density of inhibitory GAD hasket cells. Similar to the analysis above, at $400 \times$ an ocular grid was positioned over the lower half of the SG and subgranular zone (a $150 \mu \mathrm{m}$ wide area) and all GAD IR cells were counted. The procedure was repeated over the length of the stratum granulosum and the estimated densities determined incorporating Abercrombie's corrections.

Visual assessment of molecular layer sprouting. Four observers independently and blindly classified the visual pattern of IR puncta and axon fibers in the molecular layer using a progressive sprouting score of 1 to 3 as described and illustrated in Figure 1. This was a quantifiable measure of the number, direction, and orientation of axon fibers, and the amount of background IR staining (i.e., darkness) was not a component of this measure. Each observer first compared the fascia dentata molecular layer in each specimen with the histologic patterns as illustrated in the reference scale (Fig. 1; i.e., 1, 2, or 3). The observer's score could be modified up or down by 0.25 increments if the pattern looked slightly more or less than the reference in Figure 1. The four individual scores were averaged into a final semiquantitative value of axon sprouting for each peptide and GAD specimen.

$G V$ molecular layer densities. As a second measure the density (darkness) of IR in the IML and OML was measured by an image analysis computer as an average of the gray value $(\mathrm{GV})$ between white $(0)$ to black (255). A monochrome CCD cantera allached to a Zeiss microscope and interfaced with a Zeiss IBAS image analysis computer was used and the luminance was uniformly maintained between measurements. The fascia dentata molecular layer between the hippocampal fissure to the stratum granulosum was imaged and digitized. The operator outlined for the computer the region of the IML and OML, and the average $\mathrm{GV}$ of the pixels in the outlined area determined. This was repeated over five different molecular layer sample sites and the data averaged into a final GV for the IML or OML. To control for the potential bias of specimens that had been individually processed and to assure that the changes in IR were not simply from diffuse changes in existing fibers, a calculated value was determined that measured the GV difference between the IML and OML. The differences in the time from tissue "death" until fixation was longer for autopsy specimens, and the background IR staining was not as consistent as the surgical specimens. Therefore, only the specimens from HS and mass lesion/idiopathic patients which had been uniformly fixed and processed were included in this analysis.

Thickness of the supragranular molecular layer. An additional measure obtained from the image computer was the average thickness (i.e., height) of the fascia dentata molecular layer. The area from the hippocampal fissure to the top of the granule cells along the blades of the fascia dentata, avoiding the bend, were outlined for the computer. Once imaged, the computer calculated a single value that measured the average thickness along the entire fascia dentata blade.

Quantified hiplocampal neuron densities. Adjacent hippocampal sections were stained with cresylecht violet (CV) for histopathologic review $(30 \mu \mathrm{m}$ thick) and cell counts $(10 \mu \mathrm{m})$. Counts were at $400 \times$ using grid morphometric techniques with Abercrombie's (1946) corrections, and the hippocampal subfields counted were based on Lorente de
Nó's (1933) classification. The subfields were the granule cells and hilar neurons of the fascia dentata, CA4, and CA3 stratum pyramidale. A word on nomenclature. Lorente de Nó originally labeled all neurons between the blades of the stratum granulosum as CA4 and a portion of CA3c. Modern neuroanatomists recognize that this fascia dentata region contains several neuron populations. One is an extension from CA3b of pyramids (i.c., CA3c and CA4) and a second is a population of hilar neurons between the subgranular zone and pyramids. This study considered CA4 the pyramids within the stratum granulosum blades which included some $\mathrm{CA} 3 \mathrm{c}$ neurons and these pyramids were distinct anatomically and morphologically from the hilar neurons. Further, our CA3 densities were equivalent to Lorente de Nó's CA3b.

The counts were performed by one person (J.K.P.) blinded to other collected data. It should be emphasized that our neuron density method is a relative estimate and not an absolute calculation of the number of hippocampal neurons. In surgical specimens it is nearly impossible to correct for changes in tissue volume, such as from fixation shrinkage and pathologic atrophy. However, neuron densities are relative estimates, neuron losses between groups of patients can be accurately determined when comparing tissue that is similarly processed, and our method is an accepted quantificd technique in human surgical studies (Babb et al., 1984a,b). Control comparison tissue $(n=10)$ consisted of autopsies of comparable ages without cerebral pathology, processed in the same manner as the surgical material, and collected within a few hours after death prior to autolysis. Previous published studies have found that autopsy controls as used in this study do not introduce artifacts that affect human neuron density measurements, and control hippocampal cell densities are statistically stable from age 2 to 55 years (Mouritzen-Dam, 1980; Babb et al., 1984a,b; Mathern et al., 1994a,b).

Data analysis. Data were entered into a database on a personal computer and analyzed using a statistical program (SUPER ANOVA version 1.1, Abacus Concepts, Inc., Berkeley, CA). Differences between the pathogenic categories and autopsies were statistically compared using an analysis of variance (ANOVA) and further compared between individual groups (at $p<0.05$ ) using the Games-Howell test that controls for multiple comparisons of unequally sized samples and of unassumed variances. Other statistical tests included analysis of covariance (ANCOVA) and $\chi^{2}$. Results were plotted with DELTAGRAPH PROFESSIONAL (DeltaPoint, Inc., Monterey, CA). Results were considered statistically significantly different at a confidence level of $p<0.05$.

\section{Results}

Clinical profile

A total of 30 cases were studied for the peptides/GAD neuron densities and molecular layer sprouting (27 surgical specimens and three comparison autopsies). All three (NPY, SS, and GAD) were examined in 23 cases $(77 \%, 20$ surgical), and the other seven cases had two of the three. By clinical IPI histories and presurgical neuroimaging, 18 patients $(67 \%)$ fit into the HS clinical pathogenic category. Of the HS cases, nine had a single prolonged or complex first seizure, five had multiple nonprolonged childhood seizures prior to the onset of their TLE, and four had nonseizure significant medical events (i.e., cerebral trauma, hypoxia; see Mathern et al., 1994b, 1995b,d). Seven HS patients $(39 \%)$ had depth electrode studies which showed that the chronic seizure onsets were in the resected mesial temporal lobe. Post-resection seizure data (minimum 1 year, average 2.4 years) were available in $15(83 \%)$ patients, and $93 \%$ were either seizure free (class $1 ; n=10$ ) or had fewer than six seizures per year (class $2 ; n=4$ ) which was a substantial improvement compared to presurgery. There were nine patients $(33 \%)$ in the mass lesion/idiopathic category. Of the seven mass lesions, three were gliomas, two were macroscopic heterotopias, and two were cavernous angiomas. Two patients had no IPIs nor mass lesions, both continued to have frequent seizures (class 3) at least 1 year after resection, and they were classified as idiopathic TLE. The mean age at habitual TLE onsets (years \pm SEM) were not different between the two pathogenic categories (HS $13.2 \pm 2.1$; mass lesion/idiopathic $13.9 \pm 2.8 ; p=0.84$ ), and the age at 

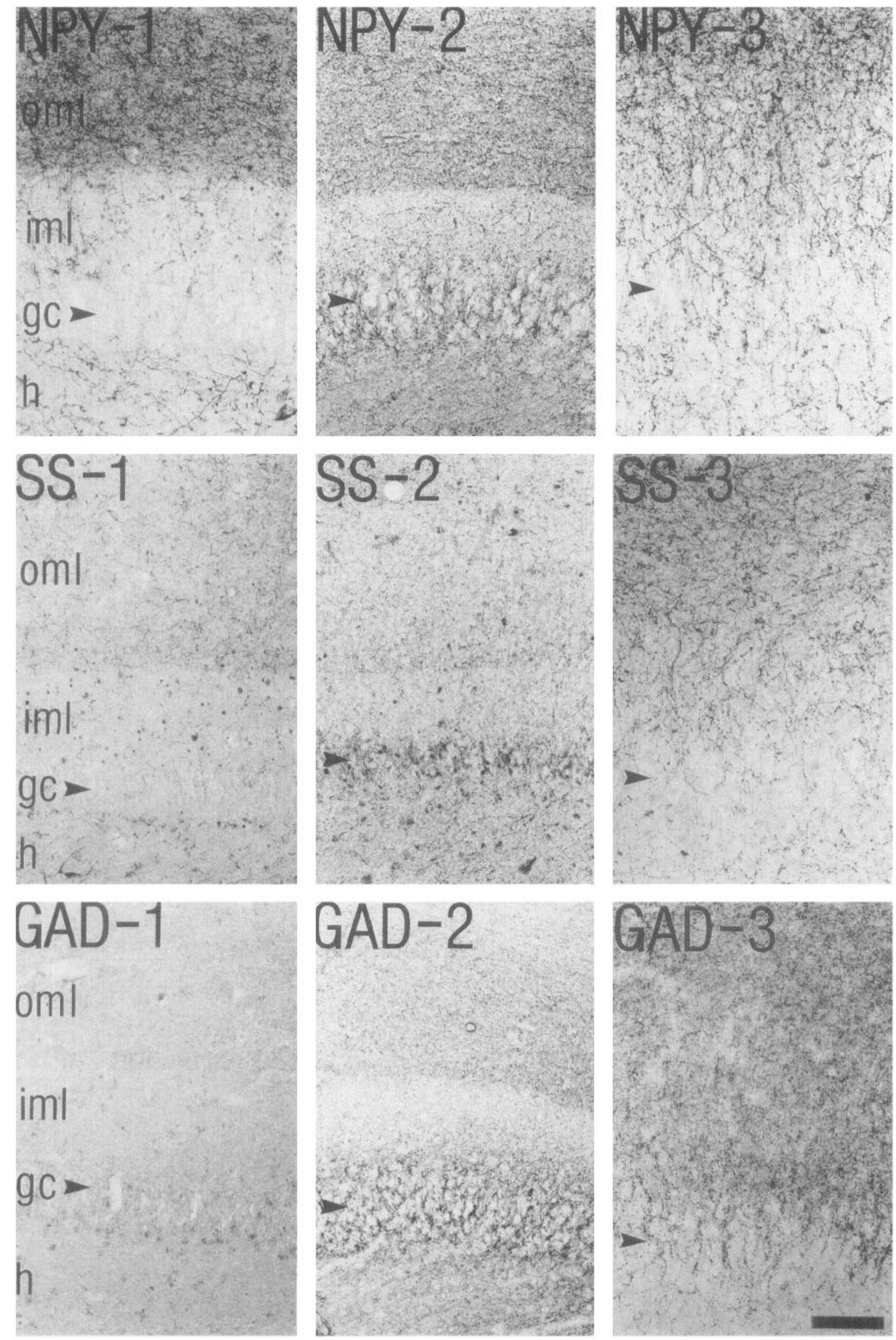

Figure 1. Photomicrographs that illustrate the semiquantitative IR visual assessments of molecular layer axon reactive synaptogenesis for NPY, $\mathrm{SS}$, and GAD. The micrographs are oriented with the outer and inner molecular layers on top (OML, IML), followed by the granule cells ( $g c$, 
surgeries were not different (HS 31.1 \pm 2.1 ; mass lesion/idiopathic $32.5 \pm 4.4 ; p=0.74)$. There were $11(41 \% ; p=\mathrm{NS})$ left-sided resections with no differences between the pathogenic categories.

\section{Neuron densities}

Figure 2 illustrates the neuron densities for the pathogenic categories and autopsies for the subfields of the fascia dentata and regio inferior. All subfields showed differences (see Fig. 2 caption), and the post hoc tests showed that compared to autopsies the pattern of neuron loss was different between HS and mass lesion/idiopathic patients. For granule cells and CA3 pyramids there were lower neuron densities in mass lesion/idiopathic and HS compared to autopsies (see*), but there were no differences between the two pathogenic categories. By contrast, hilar neurons and CA4 pyramid densities showed fewer neurons in HS compared to autopsies and mass lesion/idiopathic (see **), but there were no differences between autopsies and mass lesion/ idiopathic.

Another way to illustrate this finding is to compare the percentage of cases where the neuron densities are less than the expected $95 \%$ confidence limits of the autopsies (two tailed; $p$ $<0.025$; Table 1). The percentage of mass lesion/idiopathic cases with neuron densities below the autopsies $95 \%$ confidence level was less for the hilus $(33 \%)$ and CA4 $(0 \%)$ regions compared to the granule cells $(55 \%)$ and CA3 pyramids $(50 \%)$. By contrast, in HS patients an average $76 \%$ of cases were less than the $95 \%$ confidence level. The number of cases with neuron densities less than the $95 \%$ limit were different between HS and mass lesion/idiopathic patients $(p<0.025)$.

Regression analyses comparing the hippocampal subficld neuron densities, controlling for the pathogenic category (ANCOVA), showed that granule cell densities correlated with CA4 ( $r$ $=0.74 ; p=0.007)$ but not hilar $(p=0.10)$ and CA3 $(p=$ $0.07)$ densities. Hilar densities correlated with CA4 $(r=0.71$; $p=0.0007)$, but not CA3 ( $p=0.17)$ densities.

\section{Hilar IR neuron densities and percentages}

Figure 3 illustrates the differences in the estimated densities (upper graph) and percentages (lower graph) of hilar NPY, SS, and GAD neurons for the pathogenic categories and autopsies. The important observations were the following. (1) Compared to au-

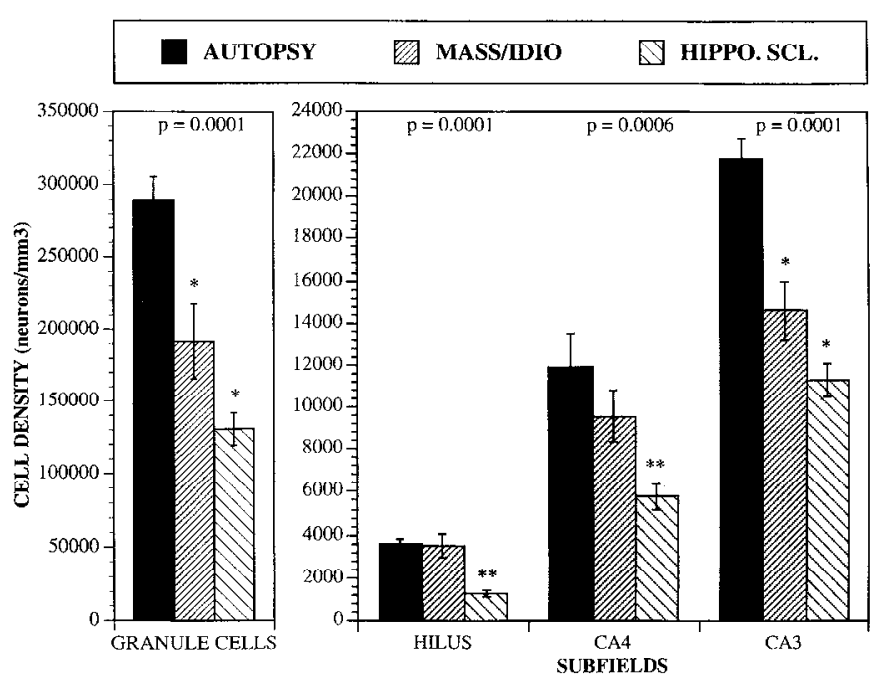

Figure 2. Graph illustrating the neuron densities in the fascia dentata and regio inferior of surgical specimens from the pathogenic categories HS and mass lesion/idiopathic compared to autopsy specimens. The neuron densities (mean \pm SEM) in the granule cells (GC) for autopsy $(289,422 \pm 16,072)$, mass lesion/idiopathic $(191,700 \pm 26,121)$, and HS $(130,600 \pm 11,380)$ were different (ANOVA; $F=13.6 ; p=$ 0.0001 ), with $\mathrm{HS}$ and mass lesion/idiopathic less than autopsy (see *; $p<0.05$; Games-Howell). Mass lesion/idiopathic and HS were not different. The fascia dentata hilar neurons for autopsy (3582 \pm 266 ), mass lesion/idiopathic $(3500 \pm 541)$, and HS (1278 \pm 175$)$ were different $(F=17.6 ; p=0.0001)$, but only HS was less than autopsy and mass lesion/idiopathic (see $* * ; p<0.05$ ). The CA4 results were similar to the pattern seen in the hilus. The densities for autopsy (11938 \pm $1528)$, mass lesion/idiopathic $(9570 \pm 1232)$, and HS $(5776 \pm 613)$ were different $(F=9.85 ; p=0.0006)$, but only HS counts were less than autopsy and mass lesion/idiopathic ( $\sec * * ; p<0.05$ ). The CA3 results were similar to the pattern for granule cells. The densities in autopsy $(21,792 \pm 947)$, mass lesion/idiopathic (14,591 \pm 1409$)$, and HS $(11,255 \pm 803)$ were different $(F=15.9 ; p=0.0001)$, with HS and mass lesion/idiopathic less than autopsy (see $* ; p<0.05$ ).

topsies, the hilar NPY densities were not different for mass lesion/idiopathic cases, but were less for HS patients $\left(^{* *}\right.$, upper graph). However, the percentages of neurons between autopsies, HS, and mass lesion/idiopathic cases were not different ( $p=$ 0.47 ; lower graph) indicating that hilar NPY neuron losses were proportional to the total amount of hilar damage. (2) Hilar SS

arrowheads), and toward the bottom the hilus $(h)$. The normal NPY pattern (NPY-l) was similar to the description by Chan-Palay et al. (1986a) and Amaral and Campbell (1986). There are very few fibers and puncta in the inner molecular layer (IML) compared to the number of immunoreactive (IR) fibers in the OML. There was a fairly distinct IR border between the IML and OML. With intermediate sprouting $(N P Y-2)$ there were slightly greater number of IR axons in the IML and some fibers appear to coarse through the gcs. However, the OML plexus was intact and the border between the IML and OML was identifiable. In severe sprouting (NPY-3) the IR axon pattern had a more uniform appearance in all molecular layers. There were more fibers and they often had a chaotic orientation, and the border between the IML and OML was very faint or absent. Notice that visually in NPY-3, the OML appears to have less IR (lighter) compared to NPY-2 consistent with the gray value measurements (see Fig. 4). The normal SS pattern (SS-I) was similar to the description of Chan-Palay (1987) and Amaral et al. (1988). There were scant IR in the IML and a faintly darker IR axon pattern in the OML that forms an semidistinct border between the IML and OML. With intermediate SS sprouting (SS-2) there are a few fibers coursing through the GCs into the IML. However, there was less IR compared to the similar score for NPY (compare NPY-2 and SS 2). In severe sprouting (SS-3) there were more fibers and puncta in the molecular layers and the border between the IML and OML was nearly absent. Notice that visually in SS-3 the molecular layers appear slightly darker compared to SS-2 (see Fig. 4). The normal GAD pattern $(G A D-I)$ was similar to the description of the normal primate fascia dentata (Babb et al., 1988a). The IR puncta were diffusely present and outlined ges. With intemitediate sprouting (GAD-2) there was greater IR puncta around ges (arrowhead) and the increased density of GAD extends into the innermost region of the IML. With severe sprouting (GAD-3) the GAD puncta extend from gcs well into the IML. Notice that visually in GAD-3 the IML appears slightly darker compared to GAD-2 (see Fig. 4). The three examples of intermediate sprouting (\#2) were from the same patient with idiopathic TLE. Seizures began without known risk factors at age 21 years and surgery was performed at age 35 years. Seizures continued following surgery at greater than 1 per month, and the overall hippocampal neuron loss averaged 16\%. The three examples of severe sprouting (\#3) were from the same patient with hippocampal sclerosis. There were frequent childhood generalized seizures from age 11 to 36 months and complex partial temporal seizures began at age 7.5 years. Surgery was at age 32 years, hippocampal neuron loss averaged $80 \%$, and the patient has been without seizures since operation. All photos are at equal magnification; scale bar, $100 \mu \mathrm{m}$. 
Table 1. Percentage of cases with neuron densities less than the 95\% confidence intervals (two tailed) of the autopsy comparison cases $(n=10)$

\begin{tabular}{llllll} 
& GCs & Hilus & CA4 & CA3 & $\begin{array}{l}\text { Av- } \\
\text { erage* }\end{array}$ \\
\hline Mass lesion/idiopathic $(n=9)$ & $55 \%$ & $33 \%$ & $0 \%$ & $50 \%$ & $35 \%$ \\
Hippocampal sclerosis $(n=18)$ & $83 \%$ & $89 \%$ & $50 \%$ & $83 \%$ & $76 \%$
\end{tabular}

${ }^{*}, \chi^{2}=5.67, p<0.025$.

densities showed a loss for HS cases but not mass lesion/idiopathic patients compared to autopsies for both the density (*, upper graph) and percentage $(*$, lower graph) measures. Thus, in HS there was a preferential loss of hilar SS neurons that exceeded the total hilar neuron loss. (3) Hilar GAD densities tended to be lower for HS and mass lesion/idiopathic cases compared to autopsies but were not statistically different ( $p=0.83$; upper graph), and numerically represented a greater proportion of hilar neurons in HS compared to mass lesion/idiopathic and autopsies ( $p=0.20$; lower graph). Further, we checked if the anterior to posterior location of the hippocampal section used to measure the neuron densities influenced the data. No statistical differences were found for NPY $(p=0.84)$, SS $(p=0.56)$, or GAD $(p=0.79)$.

\section{Inhibitory GAD basket-like cells}

In the lower half of the stratum granulosum and infragranular zone, a $150 \mu \mathrm{m}$ region, the density of GAD neurons per cubic millimeter for autopsies $(697 \pm 292)$ were numerically greater than mass lesion/idiopathic $(438 \pm 214)$, and HS (343 \pm 92$)$ patients but were not statistically different $(p=0.49)$. Further, the percentage of GAD basket-like cells compared to the total number of fascia dentata GAD neurons (hilus plus basket-like neurons) for autopsies $(65.8 \pm 11.0 \%)$, mass lesion/idiopathic $(52.6 \pm 11.5 \%)$, and HS $(49.6 \pm 6.6 \%)$ were not different $(p$ $=0.61)$.

\section{Molecular layer thickness}

The average thickness $(\mathrm{mm} \pm \mathrm{SEM}$ ) of the fascia dentata molecular layer for autopsies $(0.488 \pm 0.045)$; mass lesion/idiopathic $(0.447 \pm 0.041)$; and HS $(0.518 \pm 0.032)$ were not different $(p=0.42)$. This indicates that despite lower granule cell neuron densities in HS patients the molecular layer height between the hippocampal fissure to the top of the granule cells was not less than the non-HS cases.

\section{Visual assessments of IR axon sprouting}

The upper graph of Figure 4 shows the differences in the averaged semiquantitative scores (mean \pm SEM) of the pattern of IR axon sprouting as illustrated in the photomicrographs of Figure 1 between the pathogenic categories and autopsies. Both peptides and GAD showed differences (see Fig. 4 caption). Post hoc comparisons indicate that each category was different from any other (see **) and the greatest sprouting scores were in HS patients. Further, if autopsy cases were not considered, there was greater IR axon sprouting in HS compared to mass lesion/idiopathic for NPY $(p=0.004)$, SS $(p=0.0004)$, and GAD $(p=$ 0.018 ).

\section{Gray values of molecular layer sprouting}

The middle graph of Figure 4 shows the mean quantitative GVs and differences between HS and the mass lesion/idiopathic cat-
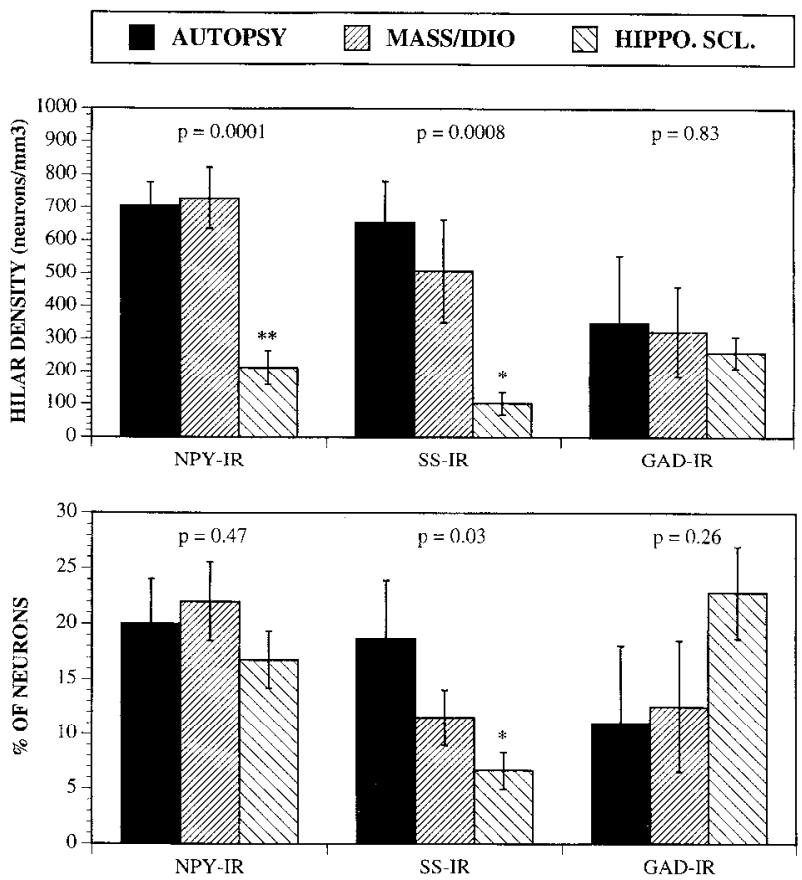

Figure 3. Graphs illustrating for autopsy, mass lesion/idiopathic, and HS the density of hilar neurons IR for NPY, SS, and GAD (upper graph), and the percentage of these IR neurons compared to the total number of hilar neurons as counted in the adjacent Nissl sections (lower graph). Upper graph, the density (mean \pm SEM) of NPY neurons for autopsy (704 \pm 75 ), mass lesion/idiopathic ( $730 \pm 93$ ), and IIS (212 $\pm 52)$ were different $(F=17.1 ; p=0.0001)$ with HS less than autopsy and mass lesion/idiopathic (see $* * ; p<0.05$ ). The SS neuron densities showed that autopsy ( $655 \pm 127)$, mass lesion/idiopathic (507 \pm 156$)$, and HS (102 \pm 34$)$ were different $(F=9.6 ; p=0.0008)$, with HS less than autopsy (see $*, p<0.05$ ). The GAD densities for autopsy $(347 \pm 208)$, mass lesion/idiopathic $(321 \pm 137)$, and HS $(258 \pm 47)$ were not different $(F=0.2 ; p=0.83)$. Lower graph, As a percentage (mean \pm SEM) of total Nissl-stained hilar neurons, NPY percentages were not different $(F=0.8 ; p=0.47)$ for autopsy $(20 \pm 4.0)$, mass lesion/idiopathic $(22 \pm 3.5)$, or HS ( $17 \pm 2.6)$. SS percentages were different $(F=4.0 ; p=0.031)$ between autopsy $(19 \pm 5.2)$, mass lesion/idiopathic $(12 \pm 2.5)$, and HS $(7 \pm 1.7)$, with HS less than autopsy (see $* ; p<0.05)$. GAD percentages were not different $(F=$ $1.4 ; p=0.259)$ between autopsy $(11 \pm 7.0)$, mass lesion/idiopathic $(12 \pm 6.0)$, and $\mathrm{HS}(23 \pm 4.2)$.

egories for the IML and OML. This was a measure of IR density in only the surgical specimens. The GV scale was from white (value of 0 ) to black (value of 255). The GVs (mean \pm SEM) ranged from $42 \pm 3$ to $105 \pm 4$. The important findings were the following. (1) Compared to mass lesion/idiopathic cases, in HS patients the NPY GVs in the OML were lighter (lower GVs; $p=0.04)$ while the IML GVs were not different $(p=0.79)$. (2) Compared to mass lesion/idiopathic cases, in HS patients the SS GVS in both the OML and IML trended toward darker IR (higher GVs) but the differences were not significant (OML $p$ $=0.054$; IML $p=0.089$ ). (3) Compared to mass lesion/idiopathic cases, in HS patients the GAD GVs in the IML were darker (higher GVs; $p=0.007$ ) while the OML GVs were not different $(p-0.82)$. Notice that for both peptides and GAD the averaged IML were always lighter (lower GV) compared to the averaged OML.

\section{$G V$ differences between the IML and $O M L$}

The averaged GVs in the middle graph of Figure 4 were direct measures from specimens collected and processed in a rigid pro- 

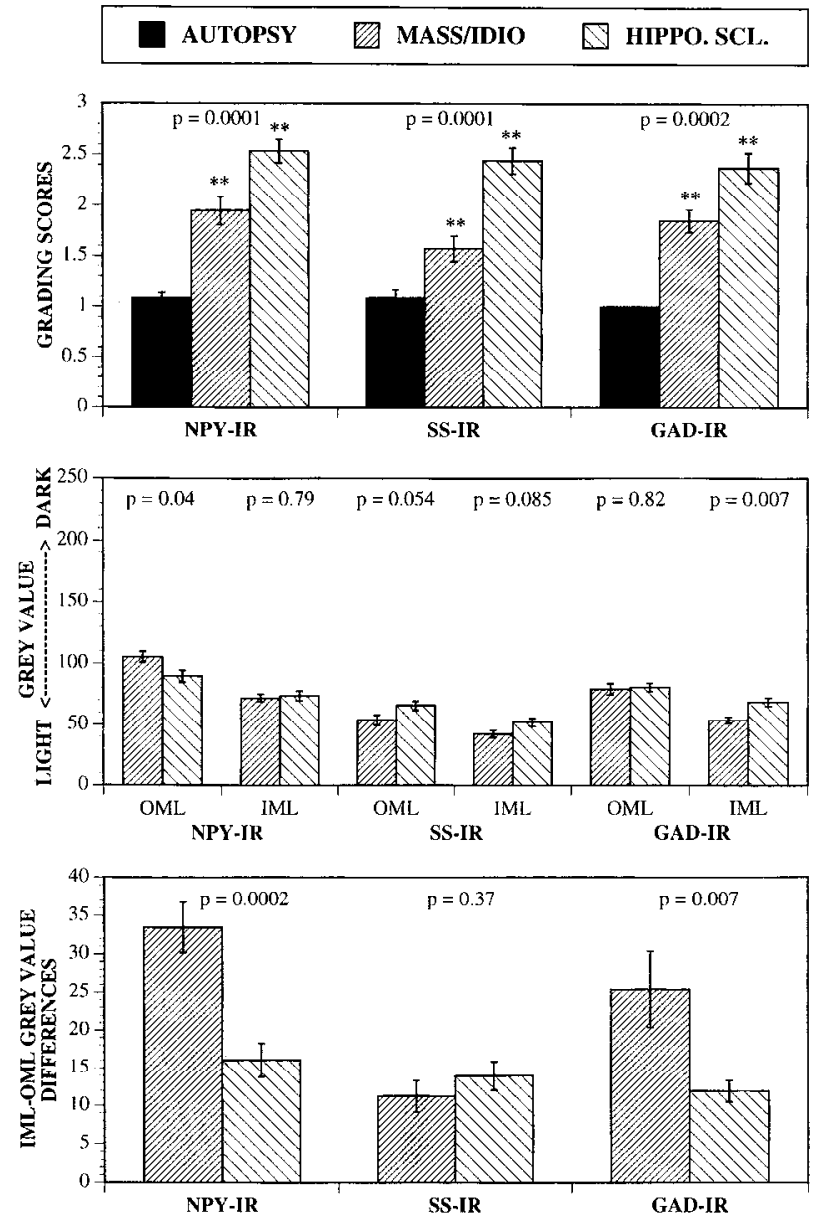

Figure 4. Graphs illustrating for NPY, SS, and GAD the differences between the pathogenic categories of (1) the averaged visual assessments of molecular layer IR sprouting as scored from Figure 1 (upper graph), (2) the averaged gray value (GV) measurements of fascia dentata inner and outer molecular layers (IML; OML; middle graph), and (3) the difference in GV measures between the IML and OML (lower graph). Upper graph, there were differences in the visual assessments (mean score \pm SEM; see Fig. 1 for details) of molecular layer IR sprouting for NPY (autopsy $1.1 \pm 0.06$; mass lesion/idiopathic $1.95 \pm$ $0.14 ; \mathrm{HS} 2.5 \pm 0.11 ; F=16.6 ; p=0.0001$ ), SS (autopsy $1.1 \pm 0.1$; mass lesion/idiopathic $1.6 \pm 0.13$; HS $2.4 \pm 0.13 ; F=16.5 ; p=$ 0.0001 ), and GAD (autopsy $1.0 \pm 0$; mass lesion/idiopathic $1.8 \perp 0.11$; HS $2.4 \pm 0.15 ; F=13.9 ; p=0.0002$ ), with each category different from the other two (see **; $p<0.05$ ). Middle graph, the GVs (mean \pm SEM; scale: white, 0 ; black, 255) showed that there were differences between peptides/GAD. The NPY GVs in the $O M L$ were lighter (lower $\mathrm{GV})$ in HS $(89 \pm 5)$ compared to mass lesion/idiopathic (105 $\pm 4 ; F$ $=4.5 ; p=0.044)$. In the IML, NPY GVs for HS $(73 \pm 4)$ and mass lesion/idiopathic $(72 \pm 3)$ were not different $(F=0.07 ; p=0.79)$. For SS. both OML and IML GVs trended toward darker IR (higher GV) in HS (OML $65 \pm 4$; IML $52 \pm 3$ ) compared to mass lesion/idiopathic (OML $54 \pm 4$; IML $42 \pm 3$, but the differences were not significant (OML $F=4.1, p=0.054$; IML $F=3.3, p=0.085$ ). By contrast, the GAD GVs in the $I M L$ were darker (higher GV) in HS $(68 \pm 4)$ than mass lesion/idiopathic $(54 \pm 3 ; F=9.3 ; p=0.007)$. In the OML, GAD-IR GVs for HS (80 13$)$ and mass lesion/idiopathic (79 \pm 5$)$ were not different $(F=0.05 ; p=0.82$ ). Notice that for each peptide and GAD the IML GVs were lower (i.e., lighter) than the OML GVs. Lower graph, the GV differences between the lighter IML (lower GVs) and darker OML (higher GVs) for the clinical pathogenic groups are illustrated. For NPY there were greater GV difference in mass lesion/ idiopathic $(33.5 \pm 3.3)$ than HS $(16.0 \pm 2.2 ; F=19.8 ; p=0.0002)$. The SS GV differences for mass lesion/idiopathic (11.2 \pm 2.1$)$ and HS $(13.9 \pm 1.9)$ were not different $(F=0.75 ; p=0.37)$. The GAD GV differences for mass lesion/idiopathic $(25.4 \pm 5.0)$ and HS (11.9 \pm 1.4) were different $(F=9.3 ; p=0.007)$. tocol. However, the individual specimens were not batched processed. A measure that reduces this variability and potential error is the GV difference between two adjacently measured regions (i.e., IML and OML). These data are illustrated in the lower graph of Figure 4. The important findings were the following. (1) For NPY $(p=0.0002)$ and GAD $(p=0.007)$ the GV differences between the IML and OML were greater in mass lesion/idiopathic compared to HS patients. This would indicate that in HS the GVs were similar throughout the molecular layer consistent with our visual assessments (see example NPY-3 and GAD-3 in Fig. 1). (2) For SS there were no differences ( $p=$ 0.37 ). Incorporating the findings of the averaged GVs (Fig. 4, middle graph), the GV difference between the IML and OML would indicate that (1) for NPY the decrease in the GV differences in HS compared to mass lesion/idiopathic means that the $O M L$ GVs were more like the IML (i.e., lighter; see Middle graph); and (2) for GAD the decrease in the GV difference in HS means that the IML was more like the OML (i.e., darker, see middle graph).

Correlation of IR molecular layer sprouting and neuron losses Single-factor correlations. The quantitative measures of IR sprouting were compared with the clinical pathogenic categories and neuron densities. The results are shown in Table 2, the sig nificant findings are in bold type and were found in $32(53 \%)$ comparisons. Correlations were more frequently found in the semiquantitative visual assessments of axon sprouting and GV differences between the IML and OML $(n=25 ; 83 \%)$ than in the averaged GVs $\left(n=7 ; 23 \% ; \chi^{2}=10.1 ; p<0.001\right)$. Further, we compared the measures of peptides/GAD sprouting to all the clinical time course variables such as duration of TLE, age at first seizure, age at IPI, etc. and one correlation was found. For HS patients the NPY GV differences between the IMI and OMI correlated with the length of the latent period (Fig. 5). In other words, in HS patients shorter latent periods correlated with lighter NPY GVs in the OML at the time of surgery.

Analyses of covariance (ANCOVA). The single factor correlations did not account for the clinical pathogenic categories when comparing neuron losses to peptides/GAD sprouting. Repeating these analyses comparing the pathogenic categories and hippocampal neuron densities to the visual assessments of IR sprouting and GV differences between the IML and OML showed several results (Table 3 ). Depending on the peptides/ GAD and the quantitative measure of IR sprouting, correlations were found with either neuron losses, especially the hilus and $\mathrm{CA} 4$, and/or the pathogenic category.

(1) Increasing amounts of visually assessed NPY axon sprouting (see Fig. 1) correlated with loss of hilar $(p=0.013)$ and CA4 ( $p=0.020)$ neurons.

(2) The NPY GV differences between the IML and OML were greater in mass lesion/idiopathic patients compared to $\mathrm{HS}$ ( $p=0.023$ ). In other words, lighter OML GVs were associated with HS compared to mass lesion/idiopathic patients. In the same column of NPY GV difference in Table 3, similar findings were suggested in the hilar and CA3 ANCOVAs but the affects were not statistically significant $(p=0.057$ and 0.07 , respectively).

(3) The visual assessments of SS axon sprouting were greater in HS compared to mass lesion/idiopathic $(p=0.002)$ and were not influenced by the GC densities $(p=0.41)$. The interactions of these two variables were also significant $(p=0.04)$.

(4) Increasing amounts of visually assessed GAD axon sprout- 
Table 2. Single-factor comparisons of molecular layer IR sprouting with neuron densities and the clinical pathogenic categories (excluded autopsy cases)

\begin{tabular}{|c|c|c|c|c|c|c|c|c|c|c|c|c|}
\hline & \multirow{2}{*}{\multicolumn{2}{|c|}{ NPY }} & \multirow{2}{*}{\multicolumn{2}{|c|}{ Somatostatin }} & \multirow{2}{*}{\multicolumn{2}{|c|}{ GAD }} & \multirow{2}{*}{\multicolumn{2}{|c|}{ NPY }} & \multirow{2}{*}{\multicolumn{2}{|c|}{ Somatostatin }} & \multicolumn{2}{|l|}{ GAD } \\
\hline & & & & & & & & & & & $\overline{\mathrm{OML}}$ & \\
\hline & Pattern & GV diff & Pattern & $\mathrm{GV}$ diff & Pattern & GV diff & OMLGV & IML GV & OMLGV & IML GV & GV & IML GV \\
\hline Path cat. & $9.99 / 0.004$ & 19.8/0.0002 & 17.3/0.0004 & $0.74 / 0.39$ & $6.76 / 0.018$ & $9.3 / 0.007$ & $4.5 / 0.044$ & $0.07 / 0.78$ & $4.2 / 0.054$ & $3.2 / 0.085$ & $0.05 / 0.82$ & $9.3 / 0.007$ \\
\hline $\mathrm{GC} \mathrm{cts}$ & $9.2 / 0.006$ & $2.1 / 0.16$ & $3.5 / 0.07$ & $0.33 / 0.57$ & $6.5 / 0.019$ & $4.2 / 0.056$ & $1.8 / 0.19$ & $0.2 \% 0.60$ & $1.4 / 0.26$ & $1.0 / 0.32$ & $0.05 / 0.83$ & $4.4 / 0.050$ \\
\hline Hilar cts & 21.4/0.0001 & $11.5 / 0.002$ & 12.2/0.002 & $0.78 / 0.38$ & $8.0 / 0.011$ & 17.1/0.0006 & $3.7 / 0.068$ & $0.01 / 0.96$ & $1.2 / 0.28$ & $0.58 / 0.45$ & $0.04 / 0.85$ & $9.7 / 0.006$ \\
\hline CA4 cts & $13.3 / 0.001$ & 8.3/0.008 & $8.7 / 0.007$ & $0.60 / 0.45$ & 17.2/0.0007 & $3.9 / 0.06$ & $2.5 / 0.12$ & $0.01 / 0.95$ & $2.3 / 0.14$ & $1.6 / 0.21$ & $0.75 / 0.40$ & $8.2 / 0.011$ \\
\hline $\mathrm{CA} 3 \mathrm{cts}$ & 6.3/0.019 & $4.4 / 0.045$ & $2.0 / 0.16$ & $1.4 / 0.24$ & $6.0 / 0.025$ & $4.8 / 0.04$ & $1.4 / 0.25$ & $0.03 / 0.86$ & $2.5 / 0.13$ & $1.2 / 0.29$ & $0.19 / 0.67$ & $6.3 / 0.022$ \\
\hline
\end{tabular}

Data are presented as $F$ value $/ p$ value. Boldface values are statistically significant at $p<0.05$.

ing correlated with loss of hilar $(p=0.023)$ and CA4 $(p=$ 0.007 ) neurons.

Finally, multivariant analyses of covariance found one result. The GAD GV differences between the IML and OML, indicating an increase in IML sprouting, correlated with GC ( $p=$ $0.015)$ and CA4 ( $p=0.02$ ) neuron losses. Overall, six comparisons found correlations between molecular layer IR axon sprouting and hippocampal neuron losses, and four comparisons found that the pathogenic category HS was associated with more peptides/GAD sprouting compared to mass lesion/idiopathic patients.

\section{Discussion}

This study found several findings that associated greater reactive synaptogenesis of fibers that were labeled for NPY, SS, and GAD in epileptogenic fascia dentata along with preferential losses of hippocampal hilar and CA4 neurons when compared with mass lesion/idiopathic patients and autopsies (nonHS). Further, our findings associates greater hilar and $C \wedge 4$ neuron losses and peptides/GAD sprouting with IPI histories. This was similar to other reports from this laboratory that have associated greater supragranular mossy fiber reactive synaptogenesis with epileptogenic human hippocampi and IPIs (Mathern et al., 1995a-c). The current findings expand upon other qualitative human hippocampal studies of peptide neuron losses and axon sprouting (de Lanerolle et al., 1989, 1992; de

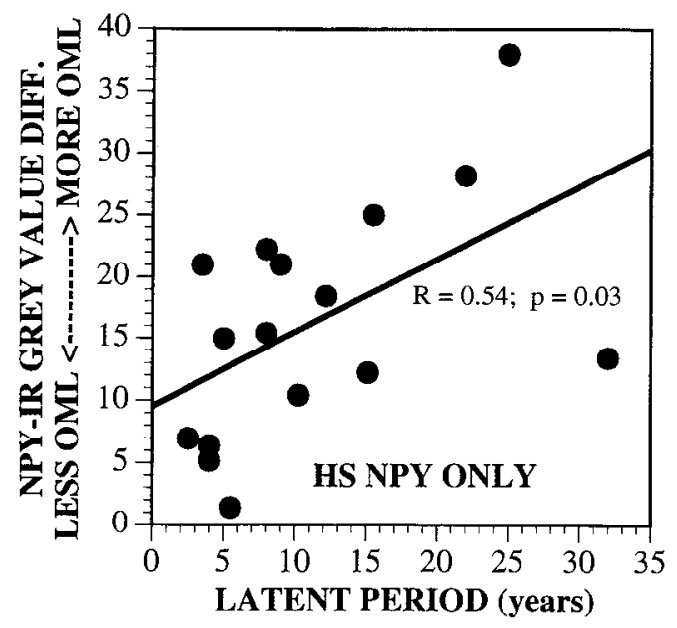

Figure 5. A correlation was found for HS patients between the length of the latent period and the GV difference between the IML and OML $(r=0.54 ; F=5.6 ; p=0.03$ ). Patients with IR lighter OMLs (i.e., less $\mathrm{GV}$ difference between the OML and IML) had shorter latent periods.
Lanerolle and Spencer, 1991), and quantitative GAD studies (Babb et al., 1989; Babb, 1992). It is worth reemphasizing that this was a retrospective study from a single epilepsy center where patients were approved for surgery based on the diagnosis that the seizure onsets were in the anterior temporal lobe, and the en bloc surgical resection was designed to remove the focus for pathologic examination. The strengths of this approach are that the patients were uniformly medically evaluated, and the independent statistical variables, such as the pathogenic categories and IPI histories, were not the primary consideration for surgery. However, our results may not be as relevant to patients with unilateral extratemporal or bilateral hemispheric seizures, may represent only some of the possible pathologic associations, and any mechanisms of pathogenesis or pathophysiology can only be inferred from our retrospective neuroanatomic study. Moreover, our measures of molecular layer IR axon sprouting were based on light microscopic analysis. We can only imply that sprouted aberrant axons form functional synapses that might contribute to epileptogenesis. However, several human studies support our assumption by showing with electron microscopy that sprouted axon terminals in the molecular layer form new synapses for SS (de Lanerolle et al., 1992), GAD (Babb et al., 1992), and Timm-stained mossy fibers (Babb et al., 1991).

It is also important to note that the expression of NPY and SS immunoreactivity in neural tissue and cerebral spinal fluid may be altered by recent seizures, antiseizure medications, and interictal electrophysiologic activity, which could potentially influence our data (Marksteiner and Sperk, 1988; Pitkänen et al., 1989; Wahlestedt et al., 1990; Sperk et al., 1992; Devinsky et al., 1993). However, we performed two different quantitative measures of IR sprouting and these techniques were (1) performed on surgical specimens that were uniformly collected and coprocessed for NPY, SS, and GAD in individual cases; (2) patients were on similar antiseizure medications; (3) they had equally frequent intractable seizures; and (4) general anesthesia during temporal resection should reduce the risk of seizures just prior to collection. Our results found differential changes of NPY, SS, and GAD GV densities and the visual pattern of axon sprouting between adjacent regions from the same specimen $(<90 \mu \mathrm{m}$; see Fig. 4), and several correlations between the amount of IR sprouting and hilar neuron losses. These findings support the notion that our experimental findings were from differences of anatomic synaptic reorganization and not from technical biases. Lastly, de Lanerolle et al. (1992) described qualitative differences in peptide synaptic plasticity and neuron losses between seizure patients with tumors and a "cryptogenic" cat- 
Table 3. Clinical pathogenic analysis of covariance (autopsy cases excluded)

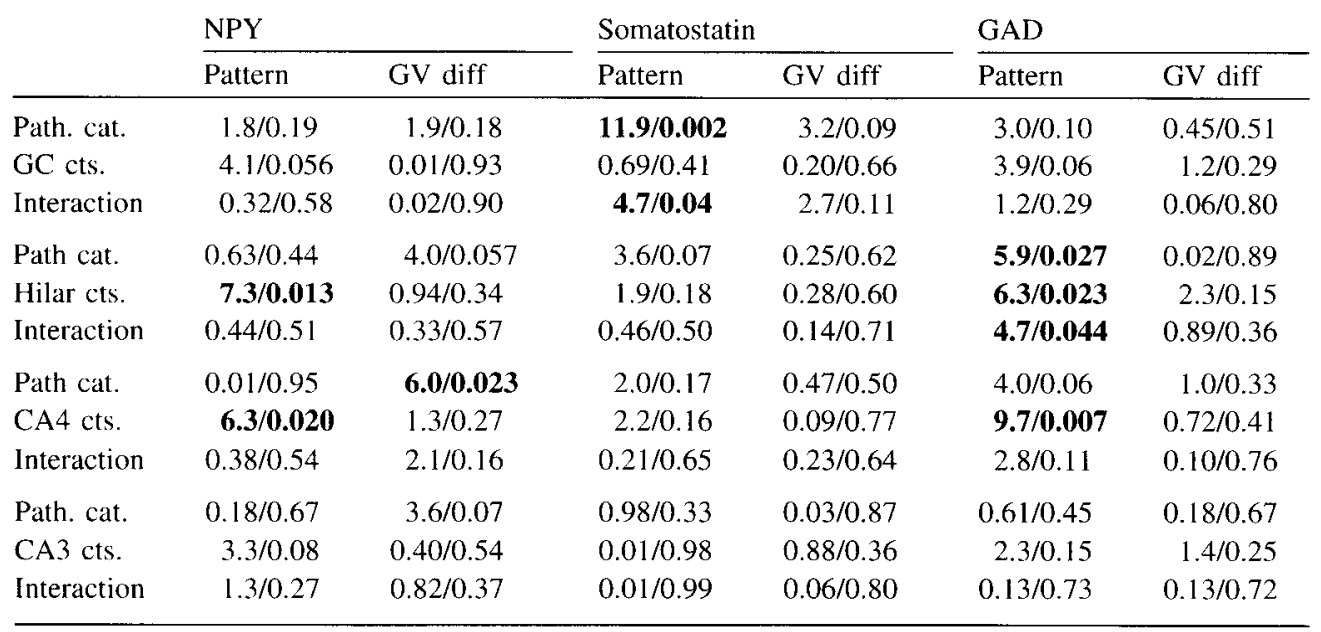

Data are presented as $F$ value/p value. Boldface values are statistically significant at $p<0.05$.

egory where the seizure onsets were in the temporal lobe based on intracerebral electrodes. Within the "cryptogenic" category were "atypical" patients whose post-resection seizure control was poor, and the qualitative patterns of peptide sprouting and cell losses were more like tumor patients. It is possible that this "atypical cryptogenic" group had clinical histories comparable to our idiopathic seizure patients.

\section{Neuroanatomic implications of peptide IR sprouting}

Our data suggest that there are important pathologic changes in HS of NPY and SS containing neurons and axon circuitry (see Fig. 6). However, interpretation of our human data requires a cautious understanding of animal experiments which are typically performed on normal hippocampi. For example, it appears that in normal rats, monkeys, and humans the anatomy of hilar NPY and SS neurons and their synaptic contacts are similar (Köhler et al., 1986; Deller and Leranth, 1990; Leranth et al., 1990) and represent a presumably inhibitory intrinsic axon system (Witter, 1993). Further, in humans approximately 30\% of hilar neurons colocalize for both NPY and SS and thus should have a common axon circuitry (Chan-Palay, 1987; Köhler et al., 1987). Likewise, in normal rats approximately $30 \%$ of hilar GAD neurons are also SS positive (Kosaka et al., 1988). Two animal studies have concluded that hilar NPY and SS neurons primarily participate in local feedforward and feedback fascia dentata circuits with little or no input of IR fibers from the major fascia dentata afferents, the fimbria fornix and perforant pathway (Bakst et al., 1986; Deller and Leranth, 1990). Hence, hilar NPY and SS neurons most likely account for the IR plexus in the fascia dentata molecular layer. Normally, dendrites of hilar NPY and SS neurons receive afferent inputs from entorhinal and commissural fibers located in the outer portions of the molecular layer, along with other synaptic contacts on their hilar soma from presumably excitatory mossy fibers and inhibitory GABAergic fibers (Fig. 6, left; Michelson and Wong, 1991; Witter, 1993; Frotscher et al., 1994). In turn, NPY and SS axons synapse on granule cells and their dendrites throughout, but primarily, in the outer molecular layer.

Based on the pattern of HS hilar neuron losses, along with an understanding of the normal NPY and SS anatomy, we can conceptually predict the probable changes in synaptic circuitry as- sociated with epileptogenic hippocampi and compare the hypothesized changes with our visual and quantitative assessments of IR sprouting (Fig. 6, right). Specifically, loss of two hilar neuron populations associated with IPIs in HS could explain the pattern of NPY and SS axon sprouting. First, the loss of mossy cells and their axons would deafferent synaptic sites in the IML (Frotscher et al., 1994). Second, loss of some of the NPY and most of the SS hilar neurons would deafferent dendritic sites in the OML. Assuming that there are not sprouted NPY and SS axons from outside the damaged hippocampus, axons from surviving hilar NPY and SS neurons could sprout to reinnervate the available deafferented granule cell dendrites in the molecular layer with a greater preference to the more abundant deafferented dendritic sites in the IML. With fewer granule cells remaining in HS, one would expect a decrease in NPY GVs such as was found in the OML; while in the IML the increased number of NPY axons in the face of fewer granule cell dendrites would not necessarily be different from normal. Further, such an interpretation could explain the diffuse and homogeneous character of immunoreactive labeling observed in the HS cases compared to mass lesion/idiopathic patients without hilar neuron losses (see Figs. 1, 4). Since the proportion of surviving hilar peptide neurons are NPY cells and the thickness of the molecular layer is the same, the sprouting of IR axons would be more apparent and measurable for NPY compared to SS, which is again consistent with our GV assessments.

Hence, one reasonable conclusion from our study would be that despite evidence of some hilar NPY and more SS hilar neuron losses associated with HS, axons IR for both peptides seem to show evidence of sprouting and probable reconnection of synapses in the fascia dentata molecular layers. These sprouted axon circuits would most likely be similar to their normal axon circuit connections with the exception that there would be a relative increased number of terminals in the IML from greater denervation there. Further, with fewer surviving hilar peptide neurons, each hilar cell may provide synaptic contacts over a larger field of the molecular layer compared to normal. Another speculative hypothesis is the possibility that hilar neurons may be capable of changing peptide expression following hippocampal injury (Sperk et al., 1992). Recall that in the normal fascia dentata there appears to be populations of hilar neurons that 
NORMAL FASCIA DENTATA

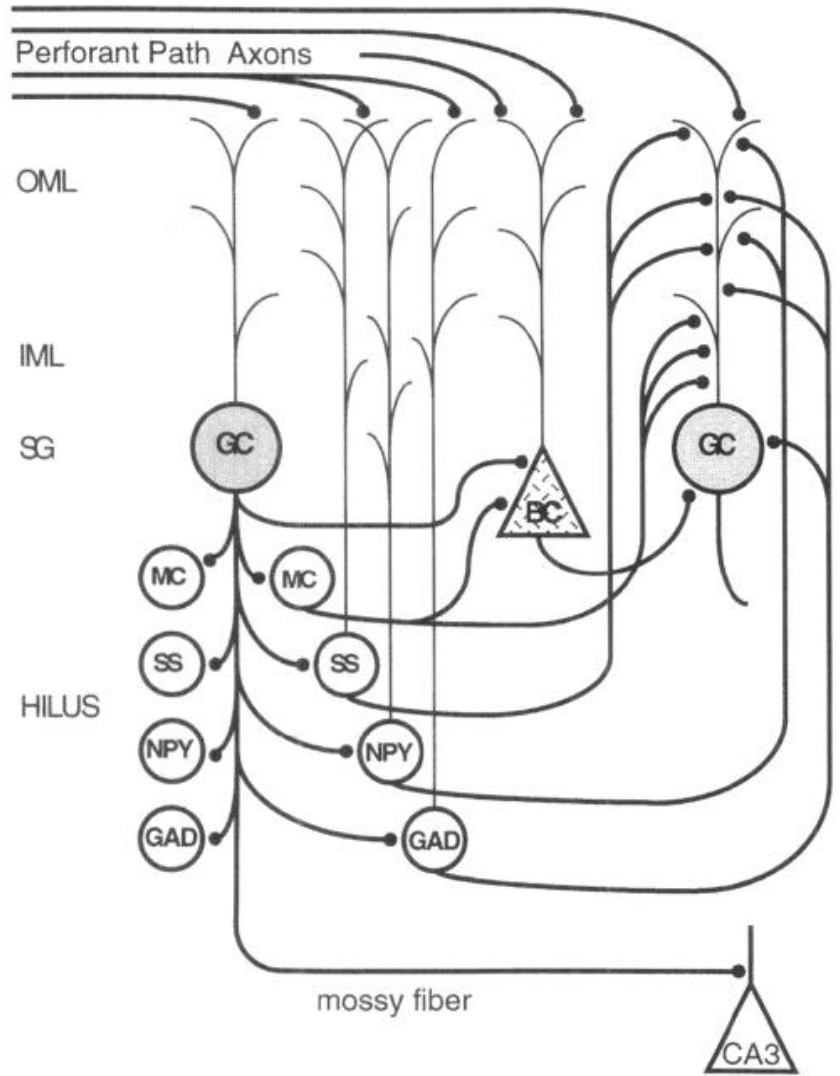

HIPPOCAMPAL SCLEROSIS

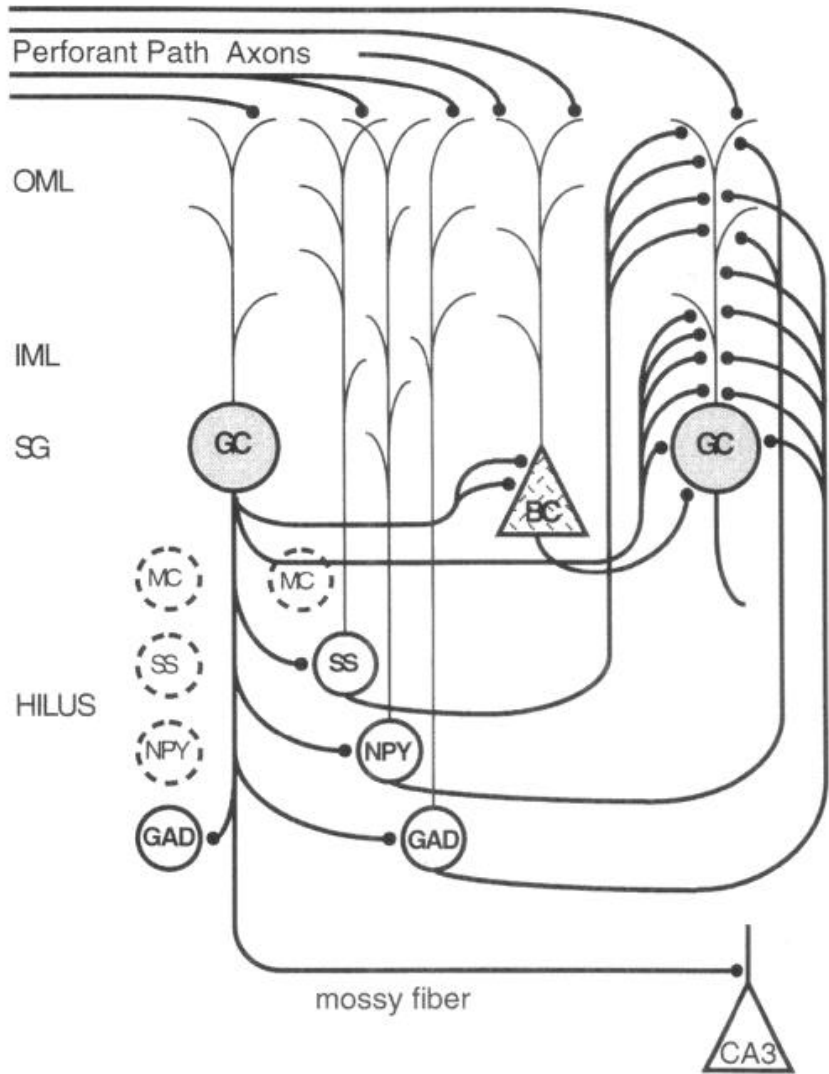

Figure 6. Scheme of the axon circuits in the normal fascia dentata (left) compared to the hypothesized axon connections that might occur in hippocampal sclerosis (right). Perforant path axons are primarily excitatory, but can contain GABA, terminate primarily on dendrites in the outer molecular layer $(O M L)$. OML dendrites include those from granule cells $(G C)$ and basket cells $(B C)$ of the stratum granulosum $(S G)$, along with hilar neurons immunoreactive for somatostatin $(S S)$, neuropeptide $\mathrm{Y}(N P Y)$, and glutamate decarboxylase $(G A D)$. The other principle hilar neuron is the mossy cell $(M C)$. Excitation of OML dendrites from the perforant path would stimulate hilar SS, NPY, and GAD neurons along with basket cells in a feedforward mechanism and their axons would modulate granule cell excitation and/or inhibition. Further, firing of granule cell axons, the mossy fibers, would also excite the same population of hilar and basket neurons in a feedback pathway. Notice that one of the afferents to basket cells is a disynaptic feedback circuit between mossy fibers and mossy cells and this is the axon circuit that the dormant basket cell hypothesis states is primarily responsible for fascia dentata inhibition to perforant path stimulation. By comparison, in hippocampal sclerosis associated with epileptogenesis there are preferential losses of mossy cells and some hilar SS and NPY neurons relative to granule cells and hilar GAD neurons (see dashed circles in hilus). Our data would be consistent with the notion that loss of these neurons and their axon connections should deafferent synaptic terminals on the inner molecular layer $(I M L)$ for MC loss, and other OML terminals from NPY and SS losses. Our findings (see Figs. 1, 4,5 ) would support the hypothesis that surviving granule cell mossy fibers sprout into the IML, and surviving hilar SS, NPY, and GAD neurons along with possibly GAD basket cells respond by sprouting axon collaterals to reinnervate deafferented terminals along the granule cell body and dendrites (right panel).

coexpress peptides and/or GAD. Hence, it is possible that surviving hilar neurons that would not normally label for NPY or SS might express immunoreactivity in hippocampal sclerosis. However, our findings would still be consistent with the notion that such surviving neurons probably would sprout reactive axons into the molecular layer.

Unfortunately, the physiologic and functional alterations of these presumably inhibitory axons that might be associated with sprouted hilar NPY and SS neurons are not readily apparent from the literature. In the normal hippocampus, Klapstein and Colmers (1993) showed that applications of NPY around pyramidal neurons presynaptically reduced excitation from stimulation of afferent axon circuits, and did not directly affect synaptically mediated inhibition. However, this affect was found in CA1 and CA3 pyramids and similar experiments on the fascia dentata granule cells did not demonstrated any physiologic affects. It has therefore been presumed that NPY acts as a modulator of neurotransmission but its exact function in the fascia dentata is unknown and the same can be said for SS (de Lanerolle and Spencer, 1991). Some evidence suggests that hilar peptide containing neurons may also contain small amounts of GABA (Halasy and Somogyi, 1993; Han et al., 1993). However, it is unknown what are the physiologic affects of small levels of GABA on SS or NPY function (Houser, 1991). Hence, it is currently impossible to accurately predict the functional consequences of NPY and SS sprouting in HS. However, we would expect based on our anatomic findings that whatever their function the physiology of these two peptides would be somewhat restored in the sprouted hippocampus, and in the area of the IML their functions might even be enhanced possibly contributing to chronic epileptogenesis (Babb et al., 1991). Obviously, additional studies will be necessary to support our predictions, but our hypothesis fits the available human anatomic data. 


\section{Neuroanatomic implications of GAD IR sprouting}

In contrast to NPY and SS, predicting in HS the possible anatomic changes in GAD circuitry is much more difficult. However, the most likely functional consequence is that GABAergic activity in the epileptogenic hippocampus is intact and probably enhanced relative to other neurotransmitters. There have been several attempts to describe the GABAergic neuroanatomy in the normal fascia dentata and the following observations show how difficult it is to study this area: (1) GABAergic neurons are a heterogeneous population of cells that occupy several areas of the fascia dentata (Ribak and Seress, 1983; Ribak, 1992), (2) there is a GABAergic component that projects with the perforant pathway into the dentate gyrus (Germroth et al., 1989), and (3) GABAergic axons form synapses with just about every dendrite, soma, and many initial axon segments in the fascia dentata (Frotscher et al., 1994). Hence, it appears that fascia dentata GABAergic neurons, especially basket cells and hilar neurons, are involved in several feedforward and feedback axon circuits onto granulc cells and other fascia dentata interneurons (Fig. 6, left).

Predicting which axon circuit from what GAD containing neuron sprouting into what new location would be extremely difficult. Yet, our two sprouting assessments for GAD would fit into a similar pattern as predicted for NPY and SS (Fig. 6, right). Loss of hilar peptide neurons and mossy cells associated with HS and IPIs would most likely deafferent synaptic sites in the molecular layer especially along the proximal granule cell dendrites (i.e., IML). The relative number of GAD neurons in the lower granule cells, infragranular, and hilar regions are not reduced in HS compared to non-HS hippocampi, and surviving GAD neurons from inside and outside the fascia dentata could sprout axon collaterals to occupy these sites. Anatomically, our sprouting assessments did not find a decrease in GAD in the OML despite fewer granule cells and an increase in GAD puncta in the IML. This would suggest that there may be a relative hyperinnervation of GAD axons in the outer molecular layer as previously concluded for GAD puncta densities around principal neurons throughout the epileptogenic human hippocampus (Babb et al., 1989). Further, in the inner molecular layer, there is probably an increase in GAD sprouting that is greater than expected.

Functionally, based on our anatomic data we would expect in the epileptogenic hippocampus that GABAergic circuitry should be intact and may even be enhanced especially along the proximal dendrites of granule cells. Moreover, several human studies support the hypothesis that GABAergic function is maintained in epileptic human hippocampi. For example, Schwartzkroin (1994) has pointed out that in human epileptic granule cells, in vitro slice experiments have all required either pharmacologic or physiologic manipulations that suppress GABAergic systems in order to demonstrate physiologic hyperexcitability (Masukawa et al., 1989, 1992; Pokorny et al., 1991). Further, there is evidence that inhibitory activity is intact in human slices (Isokawa et al., 1989; Knowles et al., 1992), and in contrast to the rat perforant path model, inhibition is enhanced with in vivo paired pulse stimulation of the perforant pathway in the epileptogenic human hippocampus (Khan et al., 1990). Yet, it is important to note that fascia dentata GABAergic physiologic activity may not be inhibitory in every circumstance. A recent study by Soltes 2 and Mody (1994) showed that activation of $\mathrm{GABA}_{\mathrm{A}}$ receptors on hilar neurons may increase firing rates.
Hence, the functional consequences of GABAergic sprouting in hippocampal sclerosis will need to await further in vivo and in vitro physiologic studies. However, it is worth reemphasizing that our anatomic data support the notion that GAD containing hilar and basket neurons are relatively spared and it appears that there may be enhancement of GAD fibers in the epileptogenic hippocampus, especially in the inner molecular layer, that was not found in nonepileptogenic hippocampi.

\section{Comparisons to the perforant path model and the dormant basket cell hypothesis}

Our anatomic data from human hippocampi show some similarities, but also a number of contrasts to the rat perforant path model and the anatomy one would expect based on the dormant basket cell hypothesis (see introductory section; Sloviter, 1991). First, similar to the rat model, there was a preferential loss of hilar SS neurons compared to NPY and GAD neurons, and this was consistent with a prior report from human hippocampi by Robbins et al. (1991). However, the preferential loss of SS applied to HS and not to mass lesion/idiopathic patients. According to the model hippocampi experiencing repeated extrahippocampal seizures over time should sustain preferential hilar SS and NPY damage (Sloviter, 1987, 1989, 1991). Hence, we would have expected that mass lesion/idiopathic cases with equal seizure histories as our HS patients should have shown similar damage to hilar SS and NPY neurons. Further, similar preferential loss of hilar SS neurons has been noted in Alzheimer's type dementia (Chan-Palay et al., 1986b; Chan-Palay, 1987) and in animal models of cerebral ischemia (Johansen, 1993; Matsuyama et al., 1993), suggesting that this pattern of hilar damage is a common finding in several pathogenic mechanisms and is not restricted to seizure associated hippocampal damage. Second, the data from the perforant path model suggested that hilar GABAergic neurons were vulnerable to seizure associated damage while inhibitory basket cells were preserved (Sloviter, 1989). Our data indicate that in the human, GAD containing neurons consistent with basket-like cells and hilar GAD neurons are both preserved relative to the loss of other fascia dentata neurons. Third, GAD fibers were anatomically more prevalent than expected in the fascia dentata molecular layer. It should be emphasized that our data cannot specifically indicate which GAD neurons contribute to GABAergic sprouting or whether basket neurons have lost mossy cell axon connections and are physiologically inactive. However, it seems likely that even if GABAergic basket cells were "dormant," then this may represent only one component of the fascia dentata feedback inhibitory axon circuitry. Based on our findings, the loss of one axon afferent to one type of inhibitory neuron is not likely to substantially reduce all GABAergic feedforward and feedback function especially in epileptogenic hippocampi with reactive synaptogenesis (see Fig. 6).

Finally, the pattern of fascia dentata and regio inferior neuron losses differs between HS and mass lesion/idiopathic patients, and this does not fit the excitotoxic perforant path modcl. Recall that according to the model, the pathogenesis of HS was that repeated seizures over time should damage first hilar followed by CA3 neurons and not granule cells. In our two pathogenic categories, HS and mass lesion/idiopathic, the averaged duration of chronic seizures were not different, and both patient groups showed loss of granule cells and CA3 pyramids compared to autopsies. This would be consistent with some excitotoxic associated damage from repeated seizure activity (see Fig. 2; Cav- 
azos et al., 1994). Yet, there was a difference in neuron losses between these pathogenic categories in hilar and CA4 neurons, which should have been the neuron populations preferentially injured even in mass lesions according to the rat model. Hence, it would appear that the pathogenic mechanisms of hippocampal hilar and CA4 neuron loss differ between hippocampal sclerosis and extrahippocampal seizure associated damage.

\section{Hypothesis of the pathogenesis and pathophysiology of hippocampal seizures}

Taken together, our human anatomic data from epileptogenic hippocampi most strongly supports the hypothesis that hippocampal seizure genesis is associated with the most severely damaged and sprouted fascia dentata. Further, the fascia dentata reorganized axons onto granule cells and their dendrites seem to include (1) monosynaptic feedback excitatory (mossy fibers), (2) feedforward and feedback inhibitory (GABAergic), and (3) feedforward and feedback, probably neuromodulator or inhibitory (NPY and SS), circuits (see Fig. 6). Hence, one hypothesis that explains the pathogenesis of hippocampal sclerosis and is based on the available anatomic and physiologic human data would be that pathologic mechanisms associated with IPIs (i.e., excitotoxic, hypoxia) probably initiate fascia dentata damage and preferentially destroy hilar mossy cells, and some NPY and SS neurons. Surviving granule cells, hilar GAD, SS, and NPY neurons sprout axon collaterals to reinnervate deafferented fascia dentata terminals and these axon fibers are likely to be both excitatory and inhibitory. There would be a latent period between the IPI and the onset of habitual temporal lobe complex partial seizures in which the fascia dentata axons reorganize and form aberrant synapses. Our finding that in HS NPY GV differences correlated with the length of the latent period is consistent with this notion (see Fig. 5). Likewise, several animal studies have shown that following hippocampal damage there is a physiologic latent period between the initial injury and the onset of spontaneous seizures. During this latent period, which can take several months in rats, axons such as mossy fibers progressively sprout into the molecular layer (Mathern et al., 1993; Mello et al., 1993; Sundstrom et al., 1993). Further, axon sprouting has been demonstrated in very young hippocampi from children with repeated severe seizures (Mathern et al., 1994a). Hence, it can be postulated that the pathophysiology of hippocampal seizures occurs because at some point progressively reorganizing excitatory and inhibitory fascia dentata axon circuits reach a critical amount and generate granule cell hyperinnervation and hypersynchrony (Dichter and Ayala, 1987; Mathern et al., 1993). By contrast, hippocampi from mass lesion/ idiopathic cases sustain less hilar seizure associated damage from extrahippocampal sources and may or may not demonstrate the amount of axon sprouting associated with hippocampal sclerosis and hence may or may not be epileptogenic. What is not known is by what mechanism would reorganized fascia dentata excitatory and inhibitory axon circuits generate physiologic seizures. As mentioned before, additional studies will be necessary to support our predictions, but our proposed hypothesis fits the currently available anatomic and physiologic data from humans with hippocampal sclerosis and intractable temporal lobe seizures.

\section{References}

Abercrombie M (1946) Estimation of nuclear population from microtome sections. Anat Rec 94:239-247.
Amaral DG, Campbell MJ (1986) Transmitter systems in the primate dentate gyrus. Human Neurobiol 5:169-180.

Amaral DG, Insausti R, Campbell MJ (1988) Distribution of somatostatin immunoreactivity in the human dentate gyrus. J Neurosci 8:3306-3316.

Anderson P, Holmqvist B, Voorhoeve PE (1966) Entorhinal activation of dentate granule cells. Acta Physiol Scand 66:448-460.

Awad IA, Rosenfeld J, Ahl J, Hahn JF, Luders H (1991) Intractable epilepsy and structural lesions of the brain: mapping, resection strategies, and seizure outcome. Epilepsia 32:179-186.

Babb TL (1991) Research on the anatomy and pathology of epileptic tissue. In: Epilepsy surgery (Luders H, ed), pp 719-727. New York: Raven.

Babb TL (1992) GABA neurons, synapses and inhibition in human hippocampal epilepsy. In: Fpilepsy and inhibition (Speckman F. Gutnick MJ, eds), pp 375-387. Munich: Urban and Schwarzenberg.

Babb TL, Brown WJ (1987) Pathological findings in epilepsy. In: Surgical treatment of the epilepsies (Engel $\mathrm{J} \mathrm{J}_{\mathrm{r}}$, ed), pp 51 l-540. New York: Raven.

Babb TL, Lieb JP, Brown WJ, Pretorius J, Crandall PH (1984a) Distribution of pyramidal cell density and hyperexcitability in the epileptic human hippocampus. Epilepsia 25:721-728.

Babb TL, Brown WJ, Pretorius J, Davenport C, Lieb JP, Crandall PH (1984b) Temporal lobe volumetric cell densities in temporal lobe epilepsy. Epilepsia 25:729-740.

Babb TL, Wilson CL, Isokawa-Akesson M (1987) Firing patterns of human limbic neurons during stereoencephalography (SEEG) and clinical temporal lobe seizures. EEG Clin Neurophysiol 66:467-482.

Babb TL, Pretorius JK, Kupfer WR, Brown WJ (1988a) Distribution of glutamate-decarboxylase-immunoreactive neurons and synapses in the rat and monkey hippocampus: light and electron microscopy. J Comp Neurol 278:121-138.

Babb TL, Kupfer WR, Pretorius JK (1988b) Recurrent excitatory circuits by 'sprouted' mossy fibers into the fascia dentata of human hippocampal epilepsy. Epilepsia 29:674.

Babb TL, Pretorius JK, Kupfer WR, Crandall PH (1989) Glutamate decarboxylase-immunoreactive neurons are preserved in human epileptic hippocampus. J Neurosci 9:2562-2574.

Babb TL, Kupfer WR, Pretorius JK, Crandall PH, Levesque MF (1991) Synaptic reorganization by mossy fibers in human epileptic fascia dentata. Neuroscience 42:35I-363.

Babb TL, Pretorius JK, Kupfer WR, Mathern GW, Crandall PH, Levesque MF (1992) Aberrant synaptic reorganization in human epileptic hippocampus: evidence for feedforward excitation. Dendron 1:725.

Bakst I, Avenadano C, Morrison JH, Amaral DG (1986) An experimental analysis of the origins of somatostatin-like immunoreactivity in the dentate gyrus of the rat. J Neurosci 6:1452-1462.

Berger MS, Ghatan S, Haglund MM, Dobbins J, Ojemann GA (1993) Low-grade gliomas associated with intractable epilepsy: seizure outcome utilizing electrocorticography during tumor resection. J Neurosurg 79:62-69.

Bruton CJ (1988) The neuropathology of temporal lobe epilepsy. New York: Oxford UP.

Cascino GD, Jack CR Jr, Parisi JE, Sharbrough FW, Schreiber CP, Kelly PJ, Trenerry MR (1993) Operative strategy in patients with MRIidentified dual pathology and temporal lobe epilepsy. Epilepsy Res 14:175-182.

Cavalheiro EA, Richie DA, Le Gal La Salle D (1982) Long-term effects of intrahippocampal kainic acid injection in rats: a method for inducing spontaneous recurrent seizures. Electroencephalogr Clin Neurophysiol 53:581-589.

Cavazos JE, Das I, Sutula TP (1994) Neuronal loss induced in limbic pathways by kindling: evidence for induction of hippocampal sclerosis by repeated brief seizures. J Neurosci 14:3106-3121.

Chan-Palay V (1987) Somatostatin immunoreactive neurons in the human hippocampus and cortex shown by immunogold/silver intensification on vibratome sections: coexistence with neuropeptide $Y$ neurons, and effects in Alzheimer-type dementia. J Comp Neurol 260: 20l-223.

Chan-Palay V, Kohler C, Haesler U, Lang W, Yasargil G (1986a) Distribution of neurons and axons immunoreactive with antisera against neuropeptide $\mathrm{Y}$ in the normal human hippocampus. J Comp Neurol 248:360-375

Chan-Palay V, Lang W, Haesler U, Kohler C, Yasargil G (1986b) Dis- 
tribution of altered hippocampal neurons and axons immunoreactive with antisera against neuropeptide $\mathrm{Y}$ in Alzheimer's-type dementia. J Comp Neurol 248:376-394.

Crandall PH (1987) Cortical resections. In: Surgical treatment of the epilepsies (Engel J Jr, ed), pp 377-404. New York: Raven.

Crandall PH, Walter RD, Rand RW (1963) Clinical applications of studies of stereotactically implanted electrodes in temporal-lobe epilepsy. J Neurosurg 20:827-840.

Davenport CJ, Brown WJ, Babb TL (1990) Sprouting of GABAergic and mossy fiber axons in dentate gyrus following intrahippocampal kainate in the rat. Exp Neurol 109:180-190.

de I anerolle NC. Spencer DD (1991) Neurotransmitter markers in human seizure foci. In: Frontiers of clinical neuroscience series, Neurotransmitters and epilepsy (Fisher RS, Coyle JT, eds), pp 201-217. New York: Wiley-Liss.

de Lanerolle NC, Kim JH, Robbins RJ, Spencer DD (1989) Hippocampal interneuron loss and plasticity in human temporal lobe epilepsy. Brain Res 495:38\%-395.

de Lanerolle NC, Brines M, Williamson A, Kim JH, Spencer DD (1992) Neurotransmitters and their receptors in human temporal lobe epilepsy. In: Epilepsy research, Suppl 7, The dentate gyrus and its role in seizures (Ribak CE, Gall CM, Mody I, eds), pp 235-250. Amsterdam: Elsevier.

Deller T, Leranth C (1990) Synaptic connections of neuropeptide Y (NPY) immunoreactive neurons in hilar area of rat hippocampus. $\mathrm{J}$ Comp Neurol 300:433-447.

Devinsky O, Emoto S, Nadi NS, Theodore WH (1993) Cerebrospinal fluid levels of neuropeptides, cortisol, and amino acids in patients with epilepsy. Epilepsia 34:255-261.

Dichter MA, Ayala GF (1987) Cellular mechanisms of epilepsy: a status report. Science 237:157-164.

Engel J Jr (1987) Outcome with respect to epileptic seizures. In: Surgical treatment of the epilepsies (Engel J Jr, ed), pp 553-571. New York: Raven.

Engel J Jr (1993) Protocols for the University of California, Los Angeles. In: Surgical treatment of the epilepsies, $2 \mathrm{~d}$ ed (Engel $\mathbf{J} \mathbf{J} \mathbf{r}$, ed), pp 743-745. New York: Ravenl.

Engel J Jr, Levesque M, Crandall PH, Shewman A, Rausch R, Sutherling W (1991) The epilepsies. In: Principals of neurosurgery (Grossman RG, ed), pp 319-358. New York: Raven.

Frotscher M, Soriano E, Misgeld U (1994) Divergence of hippocampal mossy fibers. Synapse 16:148-160.

Germroth P, Schwerdtfeger WK, Buhl EH (1989) GABAergic neurons in the entorhinal cortex project to the hippocampus. Brain Res 494: 187-192.

Goldring S, Gregorie GM (1986) Experience with gliomas and lesions which mimic them in patients with chronic seizure disorders, Part II. Clin Neurosurg 33:43-70.

Halasy K, Somogyi P (1993) Subdivisions in the multiple GABAergic innervation of granule cells in the dentate gyrus of the rat hippocampus. Eur J Neurosci 5:411-429.

Han ZS, Buhl EH, Lörinczi Z, Somogyi P (1993) A high degree of spatial selectivity in the axonal and dendritic domains of physiologically identified local-circuit neurons in the dentate gyrus of the rat hippocampus. Eur J Neurosci 5:395-410.

Houser CR (1991) GABA neurons in seizure disorders: a review of immunocytochemical studies. Neurochem Res 16:295-308.

Houser CR, Miyashiro JE, Swartz BE, Walsh GO, Rich JR, DelgadoEscuete AV (1990) Altered patterns of dynorphin immunoreactivity suggest mossy fiber reorganization in human hippocampal epilepsy. J Neurosci 10:267-282.

Isokawa-Akesson M, Wilson CL, Babb TL (1989) Inhibition in synchronously firing human hippocampal neurons. Epilepsy Res 3:236247.

Johansen FF (1993) Interneurons in rat hippocampus after cerebral ischemia. Acta Neurol Scand 150[Suppl 8]:1-32.

Khan SU, Wilson CL, Engel J Jr, Levesque MF (1990) Contrasting changes in EPSP slope of associational and perforant pathways in epileptic patients. Epilepsia 31:651.

Klapstein GJ, Colmers WF (1993) On the sites of presynaptic inhibition by neuropeptide $Y$ in rat hippocampus in vitro. Hippocampus $3: 103-112$.

Knowles WD, Awad IA, Nayel MH (1992) Differences of in vitro electrophysiology of hippocampal neurons from epileptic patients with mesiotemporal sclerosis versus structural lesions. Epilepsia 33: 601-609.

Köhler C, Eriksson LG, Davies S, Chan-Palay V (1986) Neuropeptide $\mathrm{Y}$ innervation of the hippocampal region in the rat and monkey brain. J Comp Neurol 244:384-400.

Köhler C, Eriksson LG, Davies S, Chan-Palay V (1987) Co-localization of neuropeptide tyrosine and somatostatin immunoreactivity in neurons of individual subfields of the rat hippocampal region. Neurosci Lett 78:1-6.

Kosaka 'I, Wu J-Y, Benoit $\mathrm{K}$ (1988) GABAergic neurons containing somatostatin-like immunoreactivity in the rat hippocampus and dentate gyrus. Exp Brain Res 71:388-398.

Laurberg S, Zimmer J (1981) Lesion-induced sprouting of hippocampal mossy fiber collaterals to the fascia dentata in developing and adult rats I Comp Neurol $200: 433-459$.

Leranth C, Malcolm AJ, Frotscher M (1990) Afferent and efferent synaptic connections of somatostatin-immunoreactive neurons in the rat fascia dentata. J Comp Ncurol 295:111-122.

Lorente de Nó R (1933) Studies on the structure of the cerebral cortex. II. Continuation of the study of the ammonic system. J Psychol Neurol 45:113-177.

Margerison JH, Corsellis JAN (1966) Epilepsy and the temporal lobes. Brain 89:499-530.

Marksteiner J, Sperk G (1988) Concomitant increase of somatostatin, neuropeptide $\mathrm{Y}$ and glutamate decarboxylase in the frontal cortex of rats with decreased seizure threshold. Neuroscience 26:379-385.

Masukawa LM, Higashima M, Kim JH, Spencer DD (1989) Epileptiform discharges evoked in hippocampal slices from epileptic patients. Brain Res 493:168 174.

Masukawa LM, Uruno K, Sperling M, O'Connor MJ, Burdette LJ (1992) The functional relationship between antidromically evoked field responses of the dentate gyrus and mossy fiber reorganization in temporal lobe epileptic patients. Brain Res 579:199-127.

Mathern GW, Kupfer WR, Pretorius JK, Babb TL, Levesque MF (1992) Onset and patterns of hippocampal sprouting in the rat kainate seizure model: evidence for progressive cell loss and neo-innervation in regio inferior and superior. Dendron 1:69-84.

Mathern GW, Cifuentes F, Leite JP, Pretorius JK, Babb TL (1993) Hippocampal EEG excitability and chronic spontaneous seizures are associated with aberrant synaptic reorganization in the rat intrahippocampal kainate model. EEG Clin Neurophysiol 87:326-339.

Mathern GW, Leite JP, Pretorius JK, Quinn B, Peacock WJ, Babb TL (1994a) Children with severe epilepsy: evidence of hippocampal neuron losses and aberrant mossy fiber sprouting during postnatal granule cell migration and differentiation. Dev Brain Res 78:70-80.

Mathern GW, Babb IL, Vickrey BG, Melendez M, Pretorius JK (1994b) Traumatic compared to non-traumatic clinical-pathologic associations in temporal lobe epilepsy. Epilepsy Res 19:129-139.

Mathern GW, Pretorius JK, Babb TL (1995a) Quantified patterns of mossy fiber sprouting and neuron densities in hippocampal and lesional seizures. I Neurosurg 82:211-219.

Mathern GW, Pretorius JK, Babb TL (1995b) Influence of the type of initial precipitating injury and at what age it occurs on course and outcome in patients with temporal lobe seizures. J Neurosurg 82:220227.

Mathern GW, Pretorius JK, Babb TL, Quinn B (1995c) Unilateral hippocampal mossy fiber sprouting and bilateral asymmetric neuron loss with episodic postictal psychosis. J Neurosurg 82:228-233.

Mathern GW, Babb TL, Vickrey BG, Melendez M, Pretorius JK (1995d) The clinical-pathogenic mechanisms of hippocampal neuron loss and surgical outcomes in temporal lobe epilepsy. Brain 118:105118.

Matsuyama T, Tsuchiyama M, Nakamura H, Matsumoto M, Sugita M (1993) Hilar somatostatin neurons are more vulnerable to ischemic insult than CA1 pyramidal neurons. J Cereb Blood Flow Metab 13: 229-234

McNamara JO (1994) Cellular and molecular basis of epilepsy. J Neurosci 14:3413-3425

Mello LEAM, Cavalheiro EA, Tan AM, Kupfer WR, Pretorius JK, Babb TL, Finch DM (1993) Circuit mechanisms of seizures in the pilocarpine model of chronic epilepsy: cell loss and mossy fiber sprouting. Epilepsia 34:985-995.

Michelson HB, Wong RKS (1991) Excitatory synaptic responses mediated by $\mathrm{GABA}_{\mathrm{A}}$ receptors in the hippocampus. Science 253:14201423 . 
Mouritzen-Dam A (1980) Epilepsy and neuron loss in the hippocampus. Epilepsia 21:617-629.

Oertel WH, Schmechel DE, Tappaz ML, Kopin IJ (1981) Production of specific antiserum to rat brain glutamic acid decarboxylase by injection of an antigen-antibody complex. Neuroscience 6:26892700.

Pitkänen A, Lepola U, Ylinen A, Kiekkinen PJ (1989) Somatostatin and $\beta$-endorphin levels in cerebrospinal fluid of nonmedicated and medicated patients with epileptic seizures. Neuropeptides 13:9-15.

Pokorny J, Schwartzkroin PA, Franck JE (1991) Physiologic and mor, hologic characteristics of granule cells from human epileptic hippocampus. Epilepsia 32[Suppl 3]:46.

Ribak CE (1992) Local circuitry of GABAergic basket cells in the dentate gyrus. In: Epilepsy research, Suppl 7, The dentate gyrus and its role in seizures (Ribak CE, Gall CM, Mody I, eds), pp 2917. Amsterdam: Elsevier.

Ribak CE, Seress L (1983) Five types of basket cell in the hippocampal dentate gyrus. A combined Golgi and electron microscopic study. J Neurocytol 12:577-597.

Ribak CE, Harris AB, Vaughn JE, Roberts E (1979) GABAergic nerve terminals decrease at sites of focal epilepsy. Science 205:211-214.

Ribak CE, Joubran C, Kesslak JP, Bakay RAE (1989) A selective decrease in the number of GABAergic somata occurs in pre-seizing monkeys with alumina gel granuloma. Epilepsy Res 4:126-138.

Robbins RJ, Brines JL, Kim JH, Adrian T, de Lanerolle N, Welsh S, Spencer DD (1991) A selective loss of somatostatin in the hippocampus of patients with temporal lobe epilepsy. Ann Neurol 29:325332

Schwartzkroin PA (1994) Cellular electrophysiology of human epilepsy. Epilepsy Res 17:185-192.

Sloviter RS (1983) "Epileptic" brain damage in rats induced by sustained electrical stimulation of the perforant path. I. Acute electrophysiological and light microscopic studies. Brain Res Bull 10:675697.

Sloviter RS (1987) Decreased hippocampal inhibition and a selective loss of interneurons in experimental epilepsy. Science 235:73-76.

Sloviter RS (1989) Chemically defined hippocampal interneurons and their possible relationship to seizure mechanisms. In: The hippocampus: new vistas (Köhler C, Chan-Palay V, eds), pp 443-461. New York: I iss.

Sloviter RS (1991) Permanently altered hippocampal structure, excit- ability and inhibition after experimental status epilepticus in the rat; the "dormant basket cell" hypothesis and its possible relevance to temporal lobe epilepsy. Hippocampus 1:41-66.

Sloviter RS (1992) Possible functional consequences of synaptic reorganization in the dentate gyrus of kainate-treated rats. Neurosci Lett 137:91-96.

Sloviter RS (1994) The functional organization of the hippocampal dentate gyrus and its relevance to the pathogenesis of temporal lobe epilepsy. Ann Neurol 35:640-654.

Sloviter RS, Damiano BP (1981) Sustained electrical stimulation of the perforant path duplicates kainate-induced electrophysiological effects and hippocampal damage in rats. Neurosci Lett 24:279-284.

Smialowski A, Smialowska M (1981) The effect of epileptiform discharges evoked by intrahippocampal injection of kainic acid on cholinergic and catecholaminergic hippocampal afferents. Neuroscience $6: 2519-2528$.

Soltesz I, Mody I (1994) Patch-clamp recordings reveal powerful GABAergic inhibition in dentate hilar neurons. J Neurosci 14:23652376.

Spencer SS, So NK, Engel J Jr, Williamson PD, Levesque MF, Spencer DD (1993) Depth electrodes. In: Surgical treatment of the epilepsies, 2d ed (Engel J Jr, ed), pp 359-376. New York: Raven.

Sperk G, Marksteiner J, Gruber B, Bellmann R, Mahata M, Ortler M (1992) Functional changes in neuropeptide $\mathrm{Y}$ - and somatostatin-containing neurons induced by limbic seizures in the rat. Neuroscience $50: 831-846$.

Sundstrom LE, Mitchell J, Wheal HV (1993) Bilateral reorganisation of mossy fibers in the rat hippocampus after a unilateral intracerebroventricular kainic acid injection. Brain Res 609:321 326.

Tauck DL, Nadler JV (1985) Evidence of functional mossy fiber sprouting in hippocampal formation of kainic acid treated rats. J Neurusci 5:1016-1022.

Vickrey BG, Hays RD, Graber J, Rausch R, Engel Jr J, Brook RH (1992) A health-related quality of life instrument for patients evaluated for epilepsy surgery. Med Care 30:299-319.

Wahlestedt C, Blendy JA, Kellar KJ, Heileg M, Widerlöv E, Ekman R (1990) Electroconvulsive shocks increase the concentration of neocortical and hippocampal neuropeptide Y (NPY)-like immunoreactivity in the rat. Brain Res 507:65-68.

Witter MP (1993) Organization of the entorhinal-hippocampal system: a review of current anatomical data. Hippocampus 3:33-44. 